NATIONAL LABORATORY

\title{
Effect of Air Oxidation on Pore Structure Development and Mechanical Properties of Nuclear Graphite
}

September 2010

\author{
Prepared by \\ Cristian I. Contescu \\ Senior Research Staff Scientist
}




\title{
DOCUMENT AVAILABILITY
}

Reports produced after January 1, 1996, are generally available free via the U.S. Department of Energy (DOE) Information Bridge.

Web site http://www.osti.gov/bridge

Reports produced before January 1, 1996, may be purchased by members of the public from the following source.

\author{
National Technical Information Service \\ 5285 Port Royal Road \\ Springfield, VA 22161 \\ Telephone 703-605-6000 (1-800-553-6847) \\ TDD 703-487-4639 \\ Fax 703-605-6900 \\ E-mail info@ntis.gov \\ Web site http://www.ntis.gov/support/ordernowabout.htm
}

Reports are available to DOE employees, DOE contractors, Energy Technology Data Exchange (ETDE) representatives, and International Nuclear Information System (INIS) representatives from the following source.

Office of Scientific and Technical Information

P.O. Box 62

Oak Ridge, TN 37831

Telephone 865-576-8401

Fax 865-576-5728

E-mail reports@osti.gov

Web site http://www.osti.gov/contact.html

This report was prepared as an account of work sponsored by an agency of the United States Government. Neither the United States Government nor any agency thereof, nor any of their employees, makes any warranty, express or implied, or assumes any legal liability or responsibility for the accuracy, completeness, or usefulness of any information, apparatus, product, or process disclosed, or represents that its use would not infringe privately owned rights. Reference herein to any specific commercial product, process, or service by trade name, trademark, manufacturer, or otherwise, does not necessarily constitute or imply its endorsement, recommendation, or favoring by the United States Government or any agency thereof. The views and opinions of authors expressed herein do not necessarily state or reflect those of the United States Government or any agency thereof. 
Next Generation of Nuclear Plants (NGNP) Program

\title{
EFFECT OF AIR OXIDATION ON PORE STRUCTURE DEVELOPMENT AND MECHANICAL PROPERTIES OF NUCLEAR GRAPHITE
}

\author{
Cristian I Contescu \\ Joe P Strizak \\ Tyler R Guldan \\ Timothy D Burchell
}

Date Published: September 2010

Prepared by

OAK RIDGE NATIONAL LABORATORY

Oak Ridge, Tennessee 37831-6283

managed by

UT-BATTELLE, LLC

for the

U.S. DEPARTMENT OF ENERGY

under contract DE-AC05-00OR22725 



\section{CONTENTS}

Page

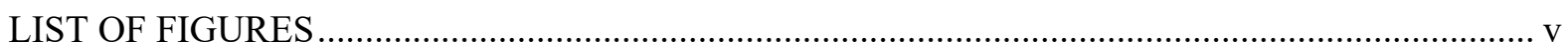

LIST OF TABLES …....................................................................................................... vii

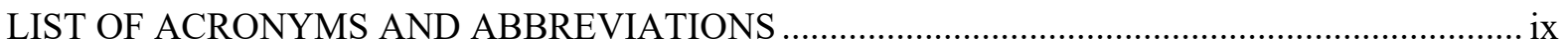

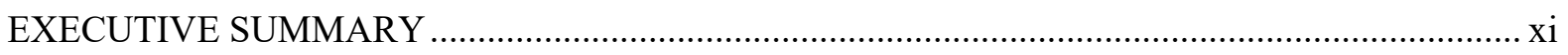

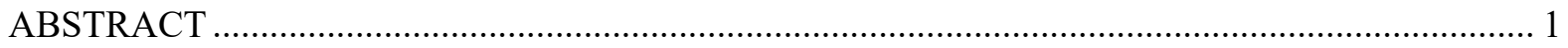

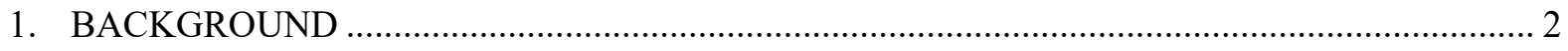

2. LITERATURE RESULTS ON THE EFFECT OF GRAPHITE OXIDATION ON

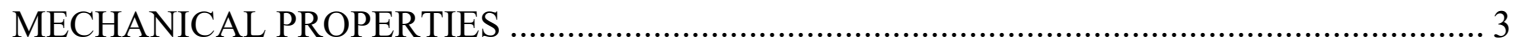

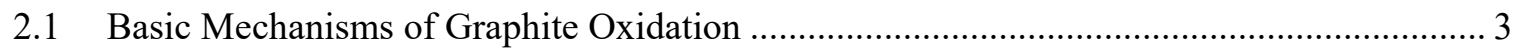

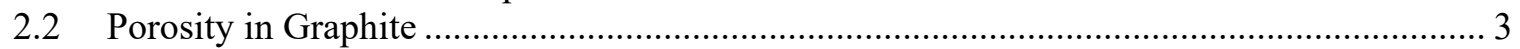

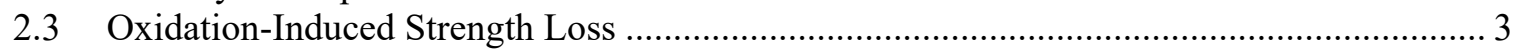

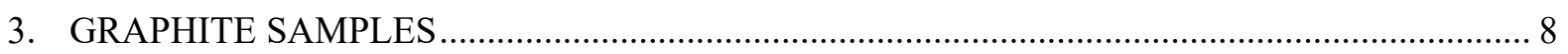

4. EFFECT OF OXIDATION ON DEVELOPMENT OF PORE STRUCTURE........................... 11

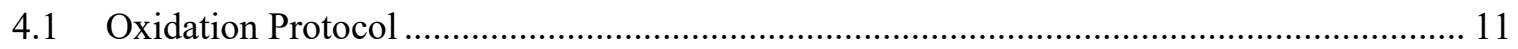

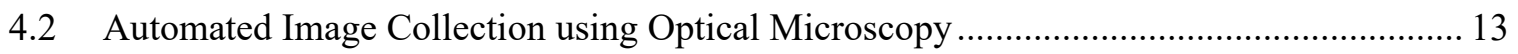

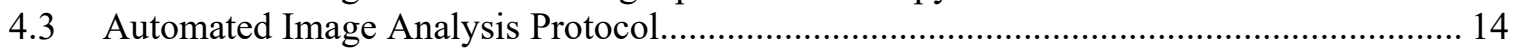

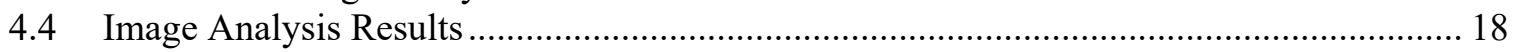

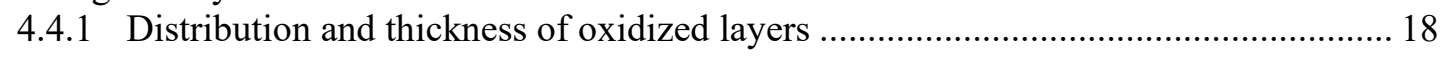

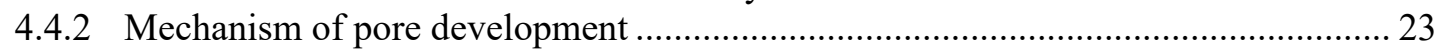

4.4.3 Effect of specimen size on oxidation layer penetration............................................ 26

5. EFFECT OF OXIDATION ON COMPRESSIVE STRENGTH LOSS .................................... 30

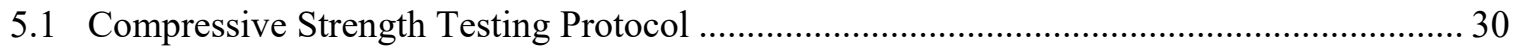

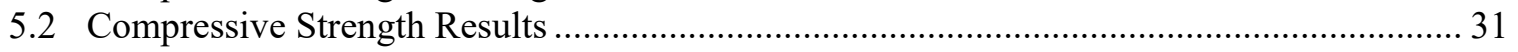

6. RELATIONSHIP BETWEEN STRENGTH LOSS AND DISTRIBUTION OF

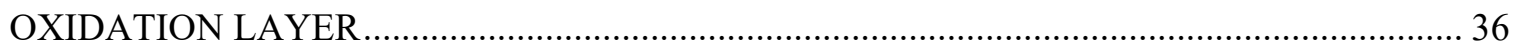

7. ADDITIONAL NON-DESTRUCTIVE PROPERTIES EXAMINATION ................................ 41

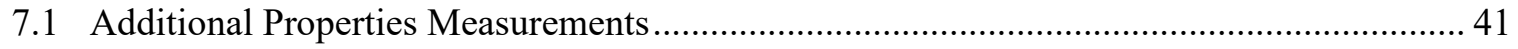

7.2 Effect of Oxidation on Young's Modulus and Shear Modulus .......................................... 41

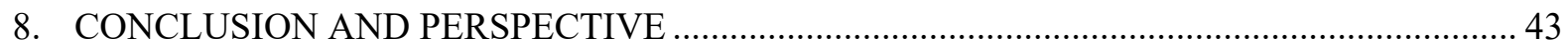

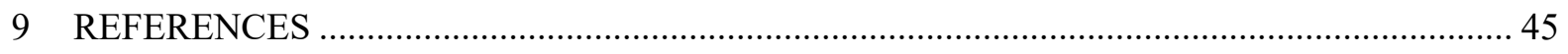

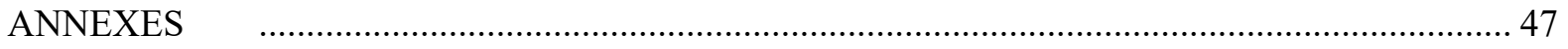


ORNL/TM-2010/197 


\section{LIST OF FIGURES}

Figure

Page

1 Effect of oxidation on compressive strength of pile-grade graphite 4

Comparison of oxidation behavior of PCEA graphite before and after purification .......... 5 Cutting diagram of PCEA graphite biller showing the position of slice \#2 which was gas-purified, and of the sub-sector (colored red) from which the specimens used in oxidation studies were further machined. 8 Photograph of oxidation apparatus used for air oxidation of graphite specimens Volume variations of PCEA graphite specimens with level of weight loss. Data for "large" specimens (25.4 mm diameter) are plotted on the left ordinate axis. Average data for "small" specimens (12.7 mm diameter) are plotted on the right ordinate axis.

Example of histogram of pixel intensities. The threshold of for pixel intensities selected for further analysis is shown on each picture.

Example of an automated image analysis sequence applied to un-oxidized specimen PW 10-3 in series of "small" graphite samples (12.7 mm diameter)................ 16 Pore volume distribution in un-oxidized PCEA graphite measured by mercury

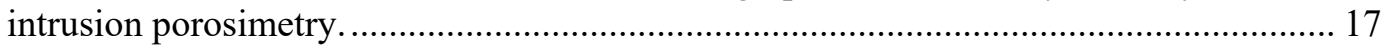

9 Development of oxidized layer in "large" PCEA graphite specimens at $600{ }^{\circ} \mathrm{C} \ldots \ldots \ldots \ldots . . . . .19$

10 Development of oxidized layer in "large" PCEA graphite specimens at $650^{\circ} \mathrm{C}$.............. 20 Development of oxidized layer in "large" PCEA graphite specimens at $750{ }^{\circ} \mathrm{C}$............... 21 Area profile histograms through the oxidized layer in "large" PCEA specimens, showing radial distribution of the oxidized layer......

Another view of images showing the structure of oxidized layer at 5-6 $\mathrm{mm}$ from surface for specimens oxidized at $600{ }^{\circ} \mathrm{C}$ in air...

Pore structure of "small" PCEA specimens (12.7 mm diameter) oriented "with grain" after oxidation in air at $600{ }^{\circ} \mathrm{C}$.

Pore structure of "small" PCEA specimens (12.7 mm diameter) oriented "with grain" after oxidation in air at $700{ }^{\circ} \mathrm{C}$. Area profile histograms across "small" PCEA specimens (12.7 mm diameter) showing spatial distribution of the oxidation layer.

Instron apparatus used for compressive strength tests.....

Compressive strength plots for pristine and oxidized PA specimens (against grain orientation), showing irregular relaxation curves and fracture aspect for "as oxidized" specimens, in contrast with shear fracture of un-oxidized sample and of oxidized specimens with $1 \mathrm{~mm}$ machined off from end faces.

20 Average values for compressive strength to failure as a function of oxidative weight loss, oxidation temperature, and grain orientation of "small PCEA test specimens.....

21 Rate of compressive strength loss versus weight loss by oxidation at 600 and 700 ${ }^{\circ} \mathrm{C}$ for "small" $\mathrm{PW}$ specimens (12.7 mm diameter) oriented "with grains". 35 Detailed view and fit (using a $4^{\text {th }}$ grade polynomial) of area pixel intensity profiles for specimens oxidized at $700{ }^{\circ} \mathrm{C}$ to three levels of weigh loss. 
23 Verification of the empirical Knudsen relationship [16] between strength loss and weight loss with local values in the oxidized layer, as obtained from the simplified mechanical model of strength-damaged annular cylinder containing an intact graphite core

24 Comparison of local properties (strength and burn-off level) in oxidized layer estimated for specimens oxidized non-uniformly at $700{ }^{\circ} \mathrm{C}$ with average properties measured for uniformly oxidized specimens at $600{ }^{\circ} \mathrm{C}$ 


\section{LIST OF TABLES}

Figure

Page

1 Impurity levels of several grades of nuclear graphite

2 Identification codes of "large" PCEA specimens (25.4 mm diameter x $25.4 \mathrm{~mm}$ length) used in oxidation studies in 2009 , showing oxidation conditions and final density (italics) after oxidation.

3 Physical properties (average values and standard deviation) before oxidation of "small" PCEA specimens (12.7 mm diameter x $25.4 \mathrm{~mm}$ length)....

4 Physical properties (average values) after oxidation at indicated temperatures of "small" specimens in series PW xx-y (PCEA purified, "with grain" orientation) analyzed in 2010

5 Results of compressive strength tests for "small" PCEA specimens $(12.7 \mathrm{~mm}$ diameter) with two different orientations oxidized at 600 and $700{ }^{\circ} \mathrm{C}$.

6 Estimated values of local density, local strength, and local burn-off in the oxidized layer (annulus) for specimens oxidized at $700{ }^{\circ} \mathrm{C}$ and three levels of burn-off, based on compressive strength tests and optical microscopy analysis of oxidized layer. Effect of oxidation temperature on the density, Young's modulus and shear modulus of "small" PA specimens (12.7 mm diameter) with "against grain" orientation 
ORNL/TM-2010/197 


\section{LIST OF ACRONYMS AND ABBREVIATIONS}

$\begin{array}{ll}\text { AIA } & \text { automated image analysis } \\ \text { ASTM } & \text { American Society for Testing and Materials } \\ \text { GDMS } & \text { glow discharge mass spectrometry } \\ \text { HTGR } & \text { high temperature gas-cooled reactor } \\ \text { INL } & \text { Idaho National Laboratory } \\ \text { NGNP } & \text { Next Generation Nuclear Plant } \\ \text { NRC } & \text { Nuclear Regulatory Commission } \\ \text { ORNL } & \text { Oak Ridge National Laboratory } \\ \text { PA } & \text { machined PCEA graphite specimens oriented "against grain" } \\ \text { PW } & \text { machined PCEA graphite specimens oriented "with grain" } \\ \text { PCEA } & \text { grade of nuclear graphite (manufactured by GrafTech International, USA) } \\ \text { PIRT } & \text { phenomena identification and ranking table } \\ \text { ppm } & \text { parts per million } \\ \text { ppmv } & \text { parts per million by volume }\end{array}$


ORNL.TM-2010/197 


\section{EXECUTIVE SUMMARY}

Chronic oxidation by impurities in the VHTR coolant gas during normal operation and acute oxidation in off-normal conditions will produce microstructural damage and changes in pore configuration in nuclear graphite, which will cause a certain decay of mechanical properties including strength loss. A knowledge gap exists regarding the relationship between oxidation behavior of graphite and its mechanical properties, as identified by the documents of the workshop on nuclear graphite research organized by ORNL and sponsored by NRC in 2009.

This report contains experimental results aimed at understanding the effect of oxidation on porosity development in PCEA-grade nuclear graphite. A statistically significant number of specimens $(25.4 \mathrm{~mm}$ length $\mathrm{x} 12.7 \mathrm{~mm}$ diameter) were oxidized in air at two temperatures $\left(600\right.$ and $\left.700{ }^{\circ} \mathrm{C}\right)$ and several levels of weight loss $(5,10,15 \%)$. The thickness, spatial distribution, and pore structure of the oxidized layer was characterized by optical microscopy coupled with automated image analysis (AIA). Mechanical properties, including compressive strength and elastic and shear moduli, were measured systematically. It was found that, at equal levels of weight loss, oxidation at $600{ }^{\circ} \mathrm{C}$ is more damaging on strength than oxidation at $700{ }^{\circ} \mathrm{C}$, although the latter is faster. Linking information obtained from image analysis and mechanical tests, an original calculation model was developed which allows estimates of local properties (i.e. density and compressive strength) in the narrow oxidized layer developed by non-uniform oxidation at high temperatures. 
ORNL.TM-2010/197

xiii 


\begin{abstract}
This document reports on continuation of R\&D activities at ORNL directed at investigating the effect of air oxidation of nuclear graphite on development and structure of porosity in the oxidized layer, and on the effect on mechanical properties of oxidized graphite. The material investigated was nuclear-grade graphite (purified PCEA graphite, from GrafTech International). Based on conclusions of previous work (ORNL/TM-2009/192), the method of analysis of the porosity in the oxidized layer based on optical microscopy coupled with automated image analysis was further improved and standardized. In addition, characterization of oxidized specimens by optical microscopy was complemented with measurements of mechanical properties (compressive strength) on a statistically significant number of specimens.

A series of cylindrical shape specimens were machined and oxidized in air at two temperatures $(600$ and $\left.700{ }^{\circ} \mathrm{C}\right)$ and at three levels of burn-off at each temperature $(5-6 \% ; 10-12 \%$ and $15-17 \%)$. The procedure used for specimen preparation and oxidation, and the oxidation equipment, were those recommended in ASTM D-7542-09. However, in order to comply with the aspect ratio requirements for specimens employed in compressive strength measurements (ASTM C-695-91) all specimens were machined with a length to diameter ratio of 2 (i.e. $25.4 \mathrm{~mm}$ height vs. $12.7 \mathrm{~mm}$ diameter) instead of 25.4 $\mathrm{mm}$ length and $25.4 \mathrm{~mm}$ diameter recommended by ASTM D-7542-09. After oxidation, mechanical properties (compressive strength) were measured on at least three samples obtained at equal oxidation conditions, while another specimen from the same lot was sectioned, mounted in resin and surface polished for optical microscopy analysis. In the latter procedure, mosaic pictures were collected of a rectangular stripe across the diameter of each specimen. These images were analyzed in order to derive the penetration depth and the pore structure in the oxidized layer as a function of oxidation temperature and weight loss level. Automated image analysis was performed using the commercial software Image Pro Plus.

The results led to the conclusion that the depth of the oxidation layer is more dependent on the oxidation temperature than on the level of weight-loss. The penetration depth of the oxidized layer is about $6 \mathrm{~mm}$ at $600{ }^{\circ} \mathrm{C}$ and about $1.5 \mathrm{~mm}$ at $700{ }^{\circ} \mathrm{C}$. Consequently, the $12.5 \mathrm{~mm}$ diameter specimens oxidized at $600{ }^{\circ} \mathrm{C}$ were completely penetrated by oxidation, while those oxidized at $700{ }^{\circ} \mathrm{C}$ had the oxidation layer restricted in a narrower band near the exposed surface. This difference was reflected by mechanical properties. A closer analysis of high magnification images in the oxidized layer revealed that oxidation attacks preferentially the binder phase in graphite, where development of fine new pores was observed. The density of new pores was higher near large pores pre-existing in graphite structure. Acute oxidation at low temperatures (e.g. $600{ }^{\circ} \mathrm{C}$ ) causes more damage on mechanical strength than high temperature oxidation $\left(700{ }^{\circ} \mathrm{C}\right)$. Although the latter is much faster, the oxidized layer is limited to a narrow zone next to the external surface, so that the core is not visibly affected. In contrast, oxidation at lower temperature, although slower, is more damaging because it penetrates deeply beyond the exposed surface.
\end{abstract}




\section{BACKGROUND}

The next generation nuclear plant (NGNP) will be a modular high-temperature gas-cooled rector (HTGR) of either prismatic or pebble-bed type. Both designs use nuclear-grade graphite as construction materials for the moderator and the core structures. The range of reactor operating temperatures are similar for the two concepts, but the irradiation level of graphite is higher for the pebble bed design. A significant challenge for construction of next generation nuclear reactors in U.S. is that the H-451 graphite qualified years ago in the United States is no longer available, and new grades of graphite must be qualified in order to replace it. The present understanding of graphite behavior is not sufficient to enable extrapolating the database of properties determined for $\mathrm{H}-451$ grade graphite to the new nuclear grade materials available now, and to the expected operating conditions of NGNP. A good understanding is needed, based on sound physical principles, of the degradation mechanism of physical, chemical, mechanical, and thermal properties of new grades of graphite that may occur during the projected life of the reactors. In particular, long time exposure to reactor chemical environment (impurities in helium coolant), under high temperature and irradiation typical of normal operating conditions, may cause chronic oxidation which will affect physical properties (including mechanical strength) of graphite. In addition, off-normal events such as air or steam ingress, although of very low probability, may severely impact the integrity of graphite in an accident scenario. The chemical resistance behavior of the new grades of graphites must be known during both normal operation and off-normal events.

In 2007 the United States Nuclear Regulatory Commission (NRC) conducted a PIRT exercise to identify phenomena that could potentially lead to accidents accompanied by release of radionuclides. The graphite PIRT panel [1] identified chronic chemical attack by impurities in the helium coolant during normal operation as a medium-importance, medium-knowledge phenomenon which may affect mechanical integrity and increase the rate of dust formation. The same panel identified strength changes induced by oxidation, temperature, and irradiation as a high-importance, medium-knowledge phenomenon. The PIRT panel emphasized the need to understand the effect of surface oxidation of core graphite on tribological behavior of graphite, and to elaborate analytical and predictive models for estimating the extent of strength loss caused by graphite oxidation and its relationship with the weight loss and oxidation conditions.

In 2009 NRC sponsored ORNL to conduct a panel workshop to review the PIRT findings, to compare them against current DOE plan, and to identify potential technology gaps. The panel [2] identified graphite oxidation as one of the specific areas which require development. It was emphasized that new specific information is needed to provide the technical base for evaluating HTGR designs for certification and licensing. In particular, the workshop panel highlighted the need to develop capability for comprehensive modeling of graphite oxidation, based on accurate knowledge of both oxidation kinetics and diffusion behavior of oxidant species within particular graphite structures. Specific oxidation rate data are needed for oxidation with low concentrations of $\mathrm{H}_{2} \mathrm{O}$ and $\mathrm{O}_{2}$ in helium in order to evaluate the long-term behavior of graphite components under normal operating conditions. This task is beyond the scope of present efforts. A second task was identified, which this report addresses. In order to develop oxidation modeling capability under accident conditions, oxidation rate constants must be measured and oxidant penetration information must be determined for conditions relevant to major accident scenarios, namely air ingress and steam ingress. Such measurements should start with laboratory scale specimens, and latter should be combined with models for structural strength loss and validation tests on larger scale components. This report responds to this task.

The report contains results of experimental work aiming at understanding the effect of oxidation on development of porosity in graphite, accompanied by changes in existing pore configuration and strength loss. This area of research was identified as a need for future work by the 2009 workshop of nuclear graphite research sponsored by NRC. [3] 


\section{LITERATURE RESULTS ON THE EFFECT OF GRAPHITE OXIDATION ON MECHANICAL PROPERTIES}

\subsection{Basic Mechanisms of Graphite Oxidation}

The dominant mechanism of graphite oxidation is controlled by the slowest process between chemical reaction, diffusion transport and mass transfer, and therefore depends strongly on temperature. [4] At low temperature oxidation is controlled by the kinetics of the chemical process, becomes diffusionlimited at high temperatures, and is limited by mass transfer in the boundary layer at very high temperatures. [5] The temperature ranges specific to each of these mechanisms are not well defined [6] and depend on sample's size and geometry [7] and on the availability (and chemical nature) of the oxidant. When the rates of oxidation are slower than those of in-pore diffusion, oxidation is under kinetic control, and the oxidant penetrates deeper into the bulk of material, leading to (almost) uniform oxidation of specimens. Although with extremely slow rate, this mechanism of oxidation may cause extensive damage of material's strength, because it extends deep into the bulk of graphite. As the temperature goes higher and the rates of reaction accelerate, diffusion rates become the controlling factor and the penetration depth of the oxidant gradually diminishes. This leads to narrow oxidation zones limited to a thin layer at the graphite exposed surface, and leaves the bulk of material practically unchanged. Consequently, in spite of evident surface damage, the mechanical strength of the core material may not be seriously affected.

\subsection{Porosity in Graphite}

Porosity is inherent to nuclear graphite; it results either from dimensional shrinking of graphite and coke, or from entrapment of gases released during thermal processing. The pore structure affects significantly the properties and behavior of nuclear graphite. Pores provide accommodation of the strain induced in graphite by irradiation. Pores also transport helium coolant gas (and the impurities that might be present in the gas) into the graphite and therefore play an important role in the oxidation behavior. Last (and most important), the pore structure plays an important role in the dynamics of fracture processes. On one hand, favorably oriented pores may be the place of crack initiation under low stress levels. On the other hand, crack propagation and growth may be influenced by the spatial relationship between large pores and pre-existing cracks. Although many types of pores with various shape and size, are present in the graphite structure, it is generally agreed that elongated, high aspect ratio pores are the most damaging to the graphite integrity [8]. Theoretical models of graphite fracture under mechanical stress [9] analyze the probability of initiation of fracture cracks and propagation of cracks to sub-critical length before catastrophic failure. When gasification of one component in the graphite structure occurs during oxidation (i.e. the binder has the weakest oxidation resistance) the basic strength properties are affected along with changes in material's microstructure and local variations in density $[10,11]$.

\subsection{Oxidation-Induced Strength Loss}

Previous research on graphite oxidation showed that mechanical properties of graphite are severely reduced by oxidation in a variety of environments $[12,13]$. It has been reported $[14,15]$ that an important reduction of strength occurs even at low oxidation weight loss. In the example shown in Figure 1 about $50 \%$ strength reduction was reported at $10 \%$ weight loss, but the strength loss versus burn-off relationship did not appear to be affected by the nature of the oxidizing gas $\left(\mathrm{O}_{2}, \mathrm{CO}_{2}, \mathrm{H}_{2} \mathrm{O}\right)$ [14]. However, it is known that the rate of oxidation and the penetration depth of the oxidized layer are controlled by the chemical nature of the oxidant species. Metal impurities present in graphite had specific catalytic activity for one gas environment, with consequential higher oxidation rates. [16] 


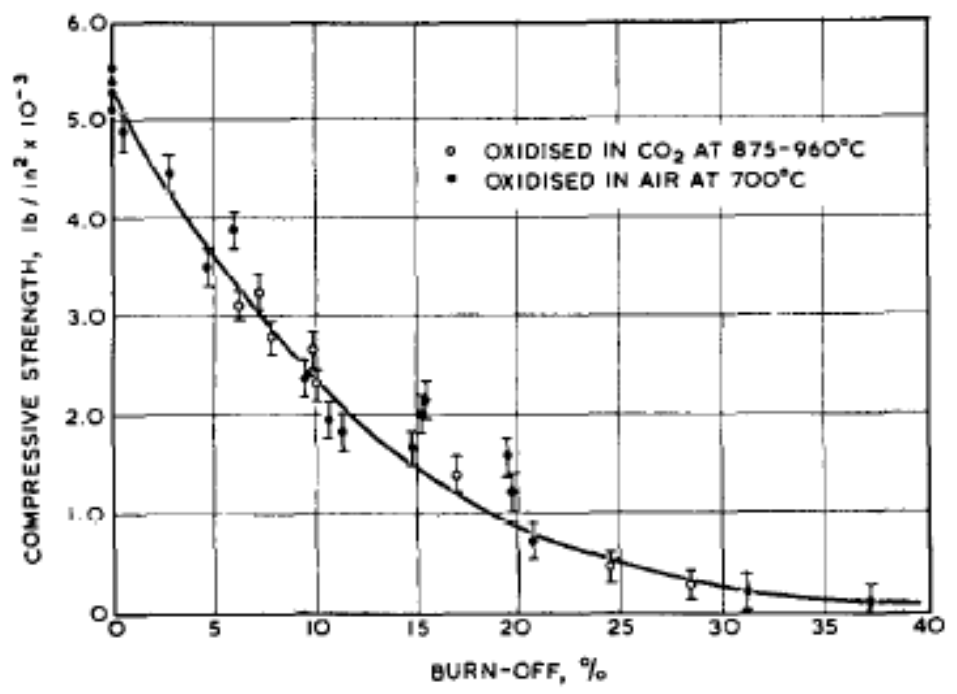

Figure 1 Effect of oxidation on compressive strength of pile-grade "A" graphite (cylindrical specimens $13 \mathrm{~mm}$ x $13 \mathrm{~mm}$ ). Adapted from ref. [14].

This behavior is also illustrated by recent oxidation rate results at ORNL obtained with PCEA graphite before and after purification. Figure 2 compares oxidation rates vs. temperature for specimens machined from as-received PCEA graphite (not purified) and from a PCEA sub-block that has been gaspurified to lower impurity levels. [17] Before purification, the major impurities in as-received PCEA graphite were $\mathrm{Al}(30-40 \mathrm{ppm}), \mathrm{Si}(30-40 \mathrm{ppm}), \mathrm{S}$ (30 ppm), Ca (30-40 ppm), V (200-500 ppm), Fe (100$200 \mathrm{ppm}) \mathrm{Ni}(60-80 \mathrm{ppm})$ and $\mathrm{Mo}(2.5 \mathrm{ppm})$. At this high content of impurities, the rates of oxidation in air were higher and had an irregular behavior, irreproducible between parallel runs and with transition from kinetic regime to pore-controlled diffusion at about $700{ }^{\circ} \mathrm{C}$. After purification the remaining impurities were $\mathrm{Si}(1.5 \mathrm{ppm}), \mathrm{S}(4.6 \mathrm{ppm}), \mathrm{V}(0.28 \mathrm{ppm})$, and $\mathrm{Mo}(0.35 \mathrm{ppm})$. Oxidation rates of purified PCEA were lower in comparable conditions, two parallel runs at four temperatures were perfectly reproducible, and the kinetics followed the Arrhenius trend (straight plots in Figure 2) at least up to 750 ${ }^{\circ} \mathrm{C}$. For comparison, Table 1 shows the impurity content of three grades of nuclear graphite reported in ref. [18]. 


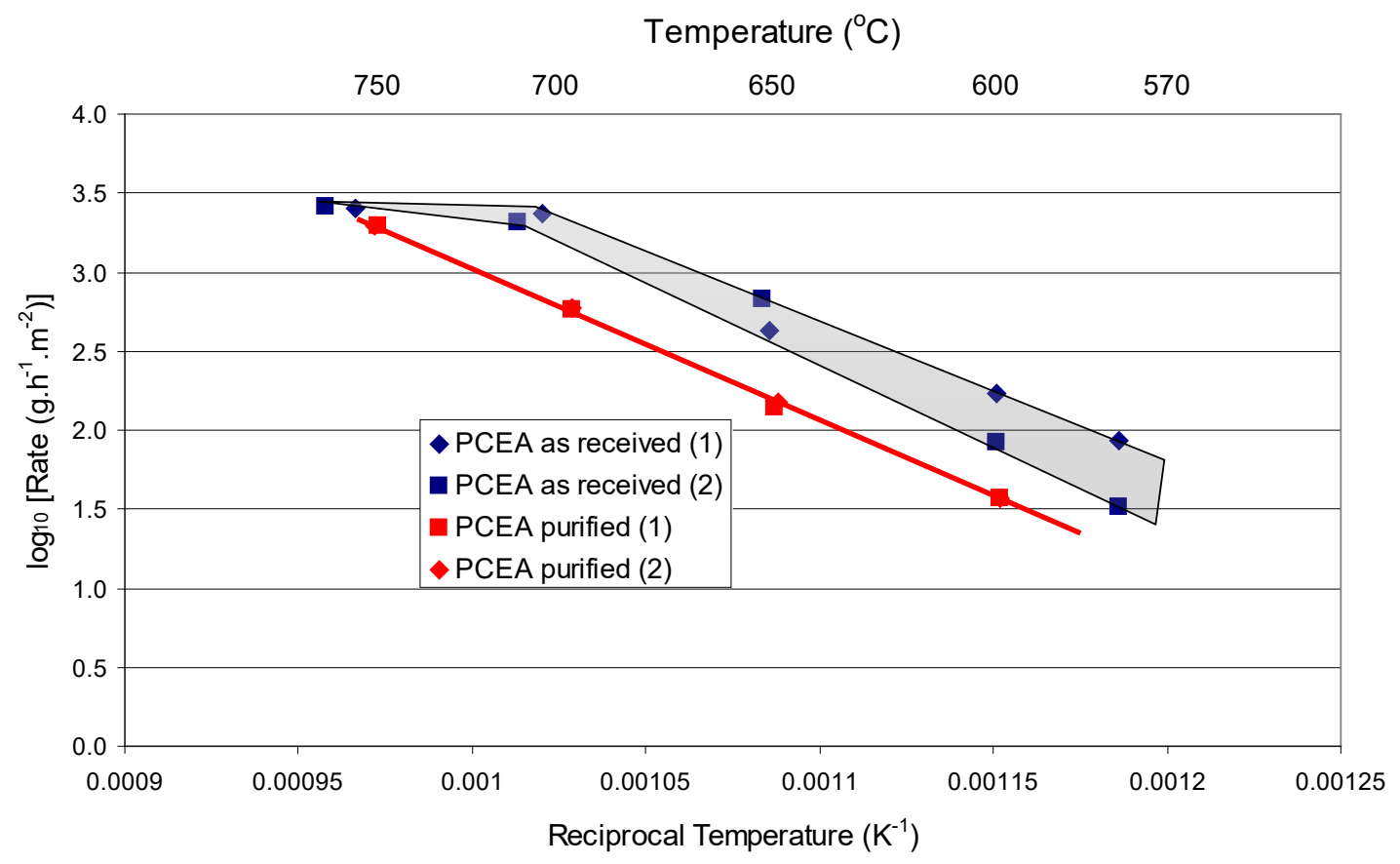

Figure 2 Comparison of oxidation behavior of PCEA graphite before and after purification (from ref. [17]).

Table 1 Impurity levels of several grades of nuclear graphite (after ref. [18])

\begin{tabular}{|c|c|c|c|}
\hline Impurities & $\begin{array}{c}\text { IG-11 } \\
(\mathrm{ppm})\end{array}$ & $\begin{array}{c}\text { PCEB } \\
(\mathrm{ppm})\end{array}$ & $\begin{array}{c}\text { PPEA } \\
(\mathrm{ppm})\end{array}$ \\
\hline $\mathrm{Fe}$ & 9.58 & 0.04 & $<0.01$ \\
$\mathrm{Ca}$ & 22.32 & $<0.05$ & 0.15 \\
$\mathrm{Mg}$ & 0.99 & $<0.05$ & $<0.05$ \\
$\mathrm{Al}$ & $<0.40$ & $<0.01$ & 0.03 \\
$\mathrm{Li}$ & 0.06 & $<0.005$ & $<0.005$ \\
$\mathrm{Sm}$ & $<0.05$ & $<0.01$ & $<0.01$ \\
$\mathrm{Gd}$ & $<0.05$ & $<0.01$ & $<0.01$ \\
$\mathrm{Co}$ & 0.20 & $<0.01$ & $<0.01$ \\
$\mathrm{Cr}$ & $<0.10$ & $<0.1$ & $<0.1$ \\
$\mathrm{Ni}$ & 8.31 & $<0.01$ & $<0.01$ \\
$\mathrm{Cd}$ & $<0.05$ & $<0.1$ & $<0.1$ \\
$\mathrm{~V}$ & 177.2 & 0.07 & $<0.005$ \\
$\mathrm{Si}$ & 0.70 & 1.3 & 6.1 \\
$\mathrm{~B}$ & 2.9 & 1.3 & 0.81 \\
$\mathrm{Ash}$ & 497 & 10 & 10 \\
\hline
\end{tabular}


Graphite may suffer structural degradation via long term oxidation by impurity gases during normal operation conditions, or during uncontrolled oxidation in an air ingress accident leading to loss of coolant.

The high rate of strength loss consistently observed during oxidation of graphite at low levels of weight loss is caused by preferential oxidation of the binder phase in graphite. The binder holds filler particles together, but the load-bearing properties are mainly due to the filler. [15] If oxidation proceeds under chemical control (Regime 1) and spreads uniformly in the bulk of machined graphite specimens with no significant dimensional changes, then the effect of burn-off on strength can be expressed [16 ] by a simple equation:

$$
\frac{\sigma}{\sigma_{o}}=m\left(\frac{\rho}{\rho_{o}}\right)^{n}=m(1-\alpha)^{n}
$$

where $\sigma$ and $\sigma_{o}$ are strength values of oxidized and un-oxidized graphite, and $\rho$ and $\rho_{\mathrm{o}}$ are corresponding density values. If the geometric size does not change during oxidation, the burn-off level (or fractional porosity) is $\alpha=1-\left(\rho / \rho_{\mathrm{o}}\right)$. In the above equation $m=1$ and $n$ is a constant. However, if oxidation occurs with significant pore-diffusion limitations (Regime 2) and surface corrosion occurs, the strength vs. burnoff relationship is better represented by the following two-term equation:

$$
\frac{\sigma}{\sigma_{o}}=m\left(\frac{\rho}{\rho_{o}}\right)^{n}+(1-m)\left(\frac{\rho}{\rho_{o}}\right)^{p}
$$

It was proposed [16] that the second, more complex relationship accounts for two parallel processes that may occur during oxidation in Regime 2: fast oxidation in open pores which causes dimensional reduction, and slower oxidation in the porosity array throughout the bulk. Surface oxidation may also cause release of loose filler particles (dust) from the oxidized surface.

Theoretical analysis of the effects of graphite oxidation on the fracture process in graphite emphasized the relationship between microstructural changes and mechanical properties. [13] Catastrophic fracture in brittle materials is quantified by the following relationship between the fracture stress $\sigma_{f}$ and critical flaw size $c$,

$$
\sigma_{f}=K_{i c} c^{-1 / 2} Y
$$

where the proportionality factor $K_{i c}$ is the critical stress intensity factor (fracture toughness) and $Y$ is a geometric constant. As evident from this equation, the decrease in strength after oxidation may be caused by a decrease in critical stress intensity $K_{i c}$, or by an increase in flaw size $c$, or by a combination of both. [19] It was found experimentally $[13,19]$ that the decrement of mechanical properties (fracture toughness, elastic modulus) introduced by the development of porosity after oxidation can be described by the empirical relationship proposed first by Knudsen [20]:

$$
Z=Z_{o} \exp (-b P)
$$

where $Z$ is the value of the mechanical property for graphite of fractional porosity $P, Z_{o}$ is a constant representing the value of property $Z$ for a notional material of zero porosity, and $b$ is a constant. This exponential decay equation applies to many mechanical properties, including critical stress intensity 
factor, critical strain energy release rate, and the work of fracture [21]. When used for graphite it is more common to express the effect of oxidation on mechanical properties using a slightly modified expression:

$$
Q=Q_{o} \exp (-b x)
$$

where $\alpha$ is the weight loss value corrected for external corrosion, and $Q_{o}$ is the value of property $Q$ for un-oxidized graphite. The latter is more accessible to measurements than the corresponding value of ideal zero-porosity graphite. [21] The corrected weight loss value is calculated from graphite density before $\rho$ and after oxidation $\left(\rho_{o}\right)$ :

$$
\alpha=1-\frac{\rho}{\rho_{o}}
$$

Changes in the oxidation conditions can substantially affect the magnitude of the decrease in the physical, elastic, and mechanical properties of graphite. For example, the toughness of graphite specimens oxidized at $500{ }^{\circ} \mathrm{C}$ was reported to be about one-half of the value for specimens oxidized at $800{ }^{\circ} \mathrm{C}$, both at $10 \%$ weight loss. [19] This observation may reflect changes of the oxidized layer thickness (and therefore of the core volume undamaged) when transition from Regime 1 to Regime 2 occurs with increase of the oxidation temperature. However the differences may be caused by more subtle reasons, related to the microstructure of the oxidized graphite.

The most significant change in the microstructure of nuclear graphite caused by oxidation is the development of narrow, slit-shaped cracks and high-aspect ratio pores in the binder phase, oriented parallel to the basal planes of graphite. This observation was made irrespective of the type of nuclear graphite investigated, either extruded (anisotropic) or molded (isotropic). [13] The shape (aspect ratio) of additional pores formed in the oxidized graphite determines the detrimental effect on the properties. Burchell et al. [22] have related the value of exponent $b$ in eq. (9) with the aspect ratio of pores in graphite. The slit-shaped pores with high-aspect ratio developed through thermal oxidation of graphite result in high values of the exponent $b$, and this is more detrimental to elastic modulus than if there had been a more uniform development of all categories of pores. In contrast, oxidation of graphite by radiolytically activated $\mathrm{CO}_{2}$ is less selective and develops pores of low aspect ratio, which cause less severe damage to elastic modulus. [22]

The effect of mechanical stress on the rate of oxidation of Stackpole 2020 graphite has been examined by Thrower and Marx. [23] They have not found any major effect of compressive stress on the rate of oxidation under their experimental conditions (either $85-175 \mathrm{ppm} \mathrm{CO}_{2}$ in $\mathrm{He}$ or $125-885 \mathrm{ppm}$ $\mathrm{H}_{2} \mathrm{O}$ in $\mathrm{He}$ at $888-908^{\circ} \mathrm{C}$ ). Similarly, no measurable effect was found when graphite was under tensile stress, in contrast with a previous report that oxidation was accelerated, allegedly, by the increase of open pores and microcracks under tensile stress. [24] No theoretical model can qualitatively explain acceleration of oxidation under tensile stress. [23] 


\section{GRAPHITE SAMPLES}

All graphite specimens covered by this report were machined from a billet of PCEA graphite purchased by ORNL in 2007 from GrafTech International Ltd. The billet was cut as shown in Figure 3. The middle slice (marked \#2 in Fig. 3) was purified by gas extraction for removal of impurities. Before purification the as-received materials contained high levels of the following impurities (results from parallel samples): $\mathrm{Na}(\sim 1 \mathrm{ppm} w \mathrm{wt}) ; \mathrm{Al}(30-40 \mathrm{ppm} w \mathrm{w}), \mathrm{Si}(30-40 \mathrm{ppm} w \mathrm{w}) ; \mathrm{S}(30 \mathrm{ppm} w \mathrm{w}) ; \mathrm{Ca}(30-$ 40 ppm wt); Ti (7 - 15 ppm wt); V (200 - 500 ppm wt); Fe (100 - 200 ppm wt); Ni (60 -80 ppm wt); Mo (2.5 ppm wt); $\mathrm{Ba}(2.5-3 \mathrm{ppm} w \mathrm{t})$. After purification, the major impurities still present were $\mathrm{Si}$ (1.5 ppm wt), S (4.6 ppm wt), P (0.22 ppm wt), V (0.28 ppm wt), and Mo (0.35 ppm wt). These results are based on analysis results by glow discharge mass spectrometry (GDMS) at Shiva Technologies in Syracuse, NY. More details on the original material, removal of impurities, and complete analysis are available from Mr. Joseph Strizak (ORNL) and in ref. [17].

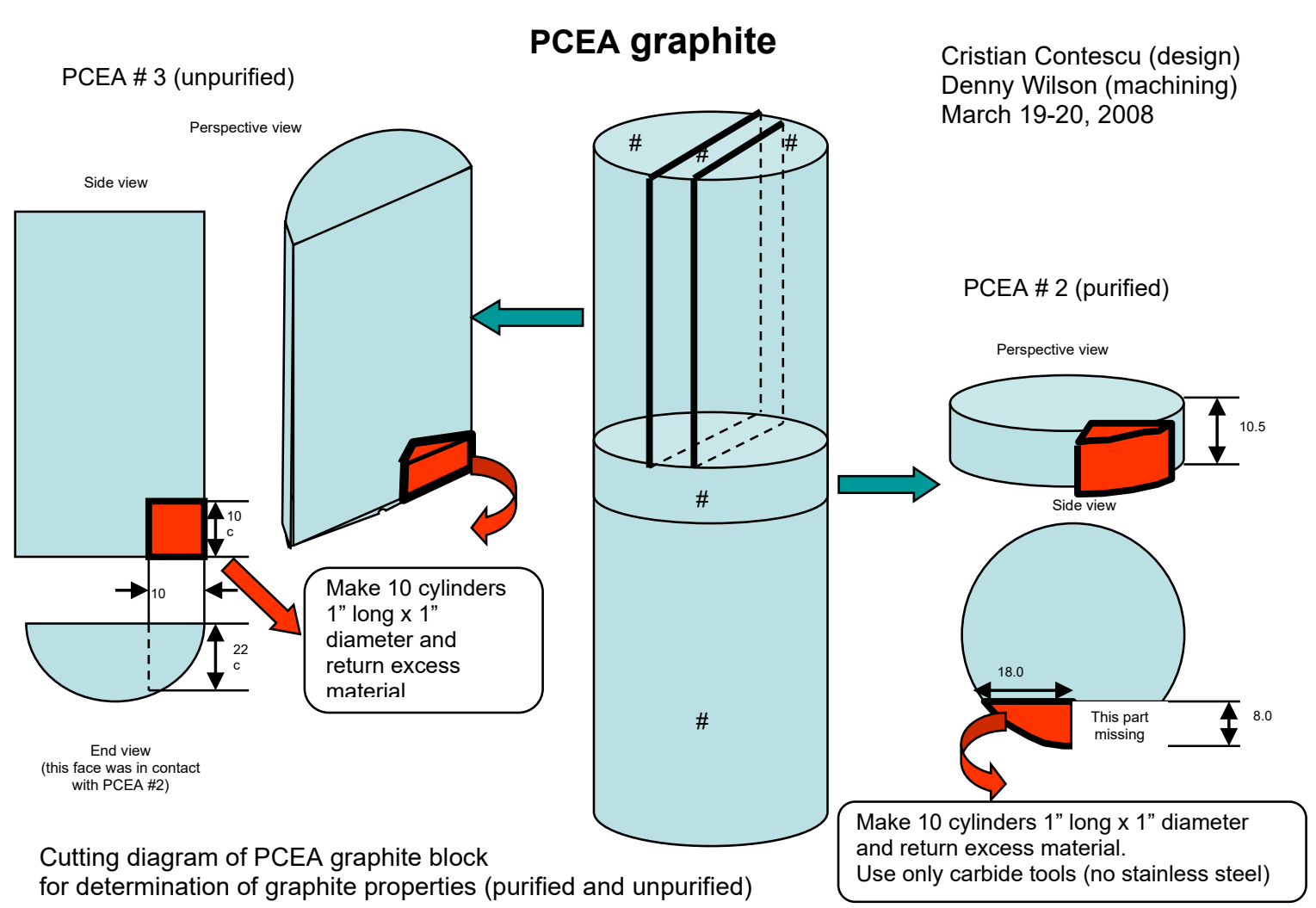

Figure 3 Cutting diagram of PCEA graphite billet showing the position of slice \#2 which was gas-purified, and of the sub-sector (colored red) from which the specimens used in oxidation studies were machined.

A sub-sector of the gas-purified slice was employed for machining of cylindrical specimens used for preliminary phase of oxidation studies and analysis of porosity development using optical microscopy and automated image analysis in 2009. [25] A number of 16 cylindrical specimens (referred to thereafter as "large" size specimens, $25.4 \mathrm{~mm}$ diameter x $25.4 \mathrm{~mm}$ length) were machined as shown in Figure 3, all having "with grain" orientation. Machining was done with iron-free tools and no lubricant was allowed. The specimens were oxidized according to the procedure recommended by ASTM D-7542 [26] at four levels of burn-off and three different temperatures. Table 2 shows identification of oxidized specimens by 
numeric codes that will be referred to later, with presentation of refined image analysis results. The table also shows the final density (in italics) of oxidized specimens.

Table2 Identification codes of "large" PCEA specimens (25.4 mm diameter x $25.4 \mathrm{~mm}$ length) used in oxidation studies in 2009 , showing oxidation conditions and final density (italics) after oxidation.

\begin{tabular}{|c|c|c|c|c|}
\hline \multirow{2}{*}{$\begin{array}{c}\text { Oxidation } \\
\text { Temperature }\end{array}$} & \multicolumn{4}{|c|}{ Burn-off degree $\%$} \\
\hline & $5 \%$ & $10 \%$ & $15 \%$ & $20 \%$ \\
\hline \multirow{2}{*}{$600^{\circ} \mathrm{C}$} & \#15 & \#14 & \#3 & \#13 \\
\hline & 1.703 & 1.61 & 1.514 & 1.524 \\
\hline \multirow{2}{*}{$650^{\circ} \mathrm{C}$} & $\# 10$ & $\# 9$ & $\# 1$ & $\# 17$ \\
\hline & 1.691 & 1.595 & 1.502 & 1.424 \\
\hline \multirow{2}{*}{$700^{\circ} \mathrm{C}$} & & & 5 & \\
\hline & & & 1.505 & \\
\hline \multirow{2}{*}{$750^{\circ} \mathrm{C}$} & \#12 & $\# 11$ & $\# 7$ & \#16 \\
\hline & 1.687 & 1.600 & 1.567 & 1.542 \\
\hline \multirow{2}{*}{ Not oxidized } & $\# 18$ & & & \\
\hline & 1.803 & & & \\
\hline
\end{tabular}

Two new series of specimens were machined in 2010 from the remaining portion of slice \#2 (see Fig. 3) of gas-purified PCEA graphite. All machining was done dry (no lubricant was allowed) with ironfree tools. The new specimens have cylindrical shape, with 2:1 ratio between length $(25.4 \mathrm{~mm})$ and diameter $(12.7 \mathrm{~mm})$. The 2:1 aspect ratio was needed in order to meet the requirements of standard test method for compressive strength of carbon and graphite (ASTM C-695) [27]. The orientation of machined specimens was both "with grain" (50 specimens labeled PW-xx-y specimens) and "against grain" (50 specimens labeled PA-xx-y specimens). The serial numbers xx-y of PA and PW specimens identifies the position of each specimen in the sub-sector of slice \#2 PCEA (purified).

Physical measurements of these specimens showed that the new lot of small size specimens was uniform. The average size and density values of specimens of each orientation are shown in Table 3. They will be referred to as "small" size specimens. 
Table 3 Physical properties (average values and standard deviation) before oxidation of "small" PCEA specimens (12.7 mm diameter x $25.4 \mathrm{~mm}$ length).

\begin{tabular}{|c|c|c|}
\hline $\begin{array}{l}\text { Series } \\
\text { Orientation } \\
\text { Properties } \\
\end{array}$ & $\begin{array}{c}\text { PA } \\
\text { against grain }\end{array}$ & $\begin{array}{c}\text { PW } \\
\text { with grain }\end{array}$ \\
\hline $\begin{array}{l}\text { Average Length }(\mathrm{mm}) \\
\text { St. dev. Length }(\mathrm{mm})\end{array}$ & $\begin{array}{r}25.377 \\
0.004 \\
\end{array}$ & $\begin{array}{r}25.360 \\
0.036 \\
\end{array}$ \\
\hline $\begin{array}{l}\text { Average Diameter }(\mathrm{mm}) \\
\text { St. dev. Diameter }(\mathrm{mm})\end{array}$ & $\begin{array}{r}12.731 \\
0.007 \\
\end{array}$ & $\begin{array}{r}12.730 \\
0.004 \\
\end{array}$ \\
\hline $\begin{array}{l}\text { Average Weight }(\mathrm{g}) \\
\text { St. dev. Weight }(\mathrm{g})\end{array}$ & $\begin{array}{ll}5.778 & \\
& 0.011 \\
\end{array}$ & $\begin{array}{r}5.809 \\
0.02 \\
\end{array}$ \\
\hline Average Density $\left(\mathrm{g} / \mathrm{cm}^{3}\right)$ & 1.806 & 1.818 \\
\hline St. dev. Density $\left(\mathrm{g} / \mathrm{cm}^{3}\right)$ & 0.005 & 0.005 \\
\hline
\end{tabular}




\section{EFFECT OF OXIDATION ON DEVELOPMENT OF PORE STRUCTURE}

\subsection{Oxidation Protocol}

The specimens were oxidized in air using the protocol recommended by ASTM D-7542. [26] The equipment used was the vertical furnace built at ORNL for graphite oxidation studies. This equipment was also used in the inter-laboratory study for verification of the above mentioned standard test method. The procedure and the method of calculation are described in details in the research report of the interlaboratory study, which is available from ASTM International as RR: D02-1663. More details are available in reference [28]. A view of the oxidation equipment is provided in Figure 4. Basically, the oxidation apparatus consists of a vertical reaction tube in a high temperature furnace. A analytical balance with under-the-scale weighing port is on top of the furnace. The graphite sample is suspended by a platinum wire in the central zone, of uniform temperature, of the furnace. The weight loss caused by oxidation is computer recorded in isothermal conditions, and the flow rate of oxidant gas (air, in this case, flowing upwards in the tube furnace) is also controlled and recorded.

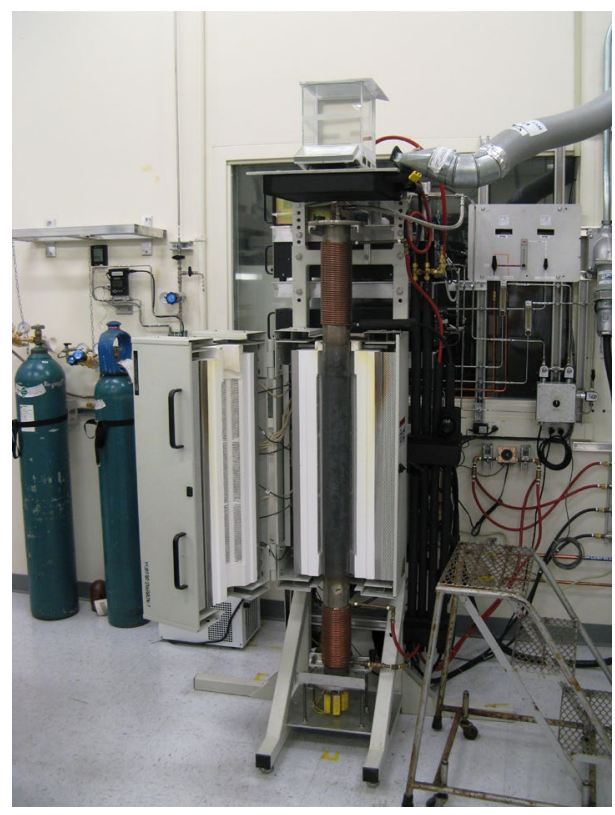

Figure 4 Photograph of oxidation apparatus used for air oxidation of graphite specimens

Oxidation of "large" graphite specimens in 2009 was done at three temperatures $(600,650$, and 750 ${ }^{\circ} \mathrm{C}$ ) and four weight loss levels (5. 10,15, and $20 \%$ ). A total of 12 oxidized specimens were obtained, labeled as shown in 3 above. Preliminary image analysis results for these specimens were reported in ref. [25]. A more in-depth analysis of the oxidized layer in these "large" specimens by optical microscopy was performed in 2010. The objective was to better measure the thickness and the distribution of the oxidized layer, and to identify the mechanism of pore developments using higher magnification images.

In 2010 "small" graphite specimens were oxidized at 600 and $700{ }^{\circ} \mathrm{C}$. The target weight loss for specimens in PW series was 5, 10 and $15 \%$ (seven specimens at each oxidation condition). For specimens in PA series the target weight loss was $10 \%$ (ten samples at each temperature). Table 4 shows average values of physical parameters measured for "small" specimens after oxidation at 600 and $700{ }^{\circ} \mathrm{C}$ 
and the maximum oxidation rates at each temperature and burn-off level. Specimen-specific results are provided in the Annex.

Most of oxidized specimens in PW series ("with grain" orientation) were used for tests of compressive strength. All of oxidized specimens in PA series were characterized by sonic velocity measurements and several of them were also tested for compressive strength. Only one of the specimens obtained at each oxidation condition in each series was analyzed by optical microscopy.

Table 4 Physical properties (average values) after oxidation at indicated temperatures of "small" specimens in series PW ("with grain" orientation) and PA ("against grain" orientation) produced and analyzed in 2010.

\begin{tabular}{ccccccccc}
\hline & & \multicolumn{7}{c}{ Average values } \\
$\begin{array}{c}\text { Oxidation } \\
\text { Temperature }\end{array}$ & $\begin{array}{c}\text { Target } \\
\text { weight } \\
\text { loss }\end{array}$ & $\begin{array}{c}\text { Final } \\
\text { weight } \\
\text { loss }\end{array}$ & Weight & Diameter & Length & Density & $\begin{array}{c}\text { Maximum } \\
\text { oxidation } \\
\text { rate }\end{array}$ & $\begin{array}{c}\text { Number } \\
\text { of } \\
\text { samples }\end{array}$ \\
\hline oC & $\%$ & $\%$ & $\mathrm{~g}$ & $\mathrm{~mm}$ & $\mathrm{~mm}$ & $\mathrm{~g} / \mathrm{cm}^{3}$ & $\mathrm{~g} \mathrm{~g}^{-1} \mathrm{~h}^{-1}$ & \\
\hline \hline$P W$ specimens & & & & & & & & \\
not oxidized & 0 & 0 & 5.816 & 12.736 & 25.370 & 1.800 & $\mathrm{n} / \mathrm{a}$ & 50 \\
600 & 5 & 5.5 & 5.504 & 12.693 & 25.373 & 1.715 & 0.0094 & 7 \\
600 & 10 & 10.2 & 5.229 & 12.699 & 25.380 & 1.628 & 0.0157 & 7 \\
600 & 15 & 15.6 & 4.903 & 12.667 & 25.360 & 1.535 & 0.1089 & 7 \\
& & & & & & & & \\
not oxidized & 0 & 0 & 5.816 & 12.736 & 25.370 & 1.800 & $\mathrm{n} / \mathrm{a}$ & \\
700 & 5 & 7.6 & 5.372 & 12.649 & 25.356 & 1.687 & 0.1089 & 7 \\
700 & 10 & 11.6 & 5.143 & 12.599 & 25.327 & 1.630 & 0.1577 & 7 \\
700 & 15 & 17.2 & 4.786 & 12.577 & 25.331 & 1.521 & 0.2059 & 7 \\
\hline$P A$ specimens & & & & & & & & \\
not oxidized & 0 & 0 & 5.778 & 12.731 & 25.377 & 1.806 & $\mathrm{n} / \mathrm{a}$ & 50 \\
600 & 10 & 10.6 & 5.189 & 12.716 & 25.389 & 1.610 & 0.0113 & 11 \\
700 & 10 & 11.9 & 5.089 & 12.696 & 25.374 & 1.585 & 0.1441 & 11 \\
\hline
\end{tabular}

Selection of oxidation conditions was driven by practical considerations. Based on oxidation results on "large" specimens, the depth of the oxidized layer formed at $600{ }^{\circ} \mathrm{C}$ is approximately $6 \mathrm{~mm}$. At this temperature, the "large" specimens $(25.4 \mathrm{~mm}$ diameter $)$ studied in 2009 were not fully penetrated by oxidation. However, the "small" specimens ( $12.7 \mathrm{~mm}$ diameter) analyzed in 2010 should be penetrated totally by the oxidant, thus producing a sample (more or less) uniformly oxidized. On the other hand, it should be expected that the "small" specimens would be oxidized only in the superficial layer at $700{ }^{\circ} \mathrm{C}$. A direct measurement of the depth of the oxidized layer was not made at $700{ }^{\circ} \mathrm{C}$ in the 2009 study. The data reported in that study showed however that the oxidized layer of "large" specimens was about 3-4 $\mathrm{mm}$ thick at $650{ }^{\circ} \mathrm{C}$ and less than $1 \mathrm{~mm}$ thick at $750{ }^{\circ} \mathrm{C}$.

Because of the smaller size the specimens studied in 2010 (1/4 of the weight of "large" specimens) shortened the time needed to achieve the same levels of weight loss, compared with the "large" specimens. This made it more difficult to accurately control the oxidation time, and accurately reproduce equal levels of weight loss for all specimens in a big lot, especially at high temperatures. For this reason, it was decided to operate at lower oxidation temperature $\left(700{ }^{\circ} \mathrm{C}\right.$, compared to $750{ }^{\circ} \mathrm{C}$ in 2009$)$.

Because of the large number of oxidized specimens needed to study mechanical properties (total about 50), it was attempted first to produce them in large batches using a muffle furnace with air circulation. However, it soon became clear that the air circulation and/or the temperature inside this muffle furnace were not uniform. After holding in the furnace a predetermined time, the lot of oxidized 
specimens was not uniform, with large variations from sample to sample. This method had to be abandoned, and the next specimens were oxidized one by one in the vertical furnace with controlled air circulation and temperature.

In the vertical furnace (Fig. 4) the final level of oxidation is controlled by cutting off the power to the furnace when weight loss reached a preselected target value. However, the air is not switched to inert gas at the same time, and oxidation continues (with slower rates) during the first part of natural cooling of the furnace. Because of that the final level of oxidation for the "small" specimens oxidized at $700{ }^{\circ} \mathrm{C}$ was slightly larger than the targeted values.

The duration of oxidation varied between large limits: At $600{ }^{\circ} \mathrm{C}$ is took about $\sim 8$ hours to get $5 \%$ weight loss and $\sim 18 \mathrm{hr}$ for $15 \%$ weight loss. At $700{ }^{\circ} \mathrm{C}$ the time needed to get $5 \%$ weight loss was less than 30 minutes, while the time for $15 \%$ weight loss was about 1 hour. As expected, oxidation rates increased with the temperature, and also with the final weight loss level. This is a known property of graphite oxidation. [6]

The variation of the volume of oxidized specimens is shown in Figure 5. Interestingly, the volume of "large" specimens did not change significantly after oxidation at 600 and $650{ }^{\circ} \mathrm{C}$, but for "small" specimens a slight decrease in volume was observed at $600{ }^{\circ} \mathrm{C}$. This indicates that some amount of surface oxidation occurs at $600{ }^{\circ} \mathrm{C}$ in "small" specimens, although that is not observed in "large" specimens. The linear decrease with weight loss at higher oxidation temperatures $\left(750^{\circ} \mathrm{C}\right.$ for "large" specimens, $700{ }^{\circ} \mathrm{C}$ for "small" specimens) indicates that oxidation occurred preferentially at the external surface.

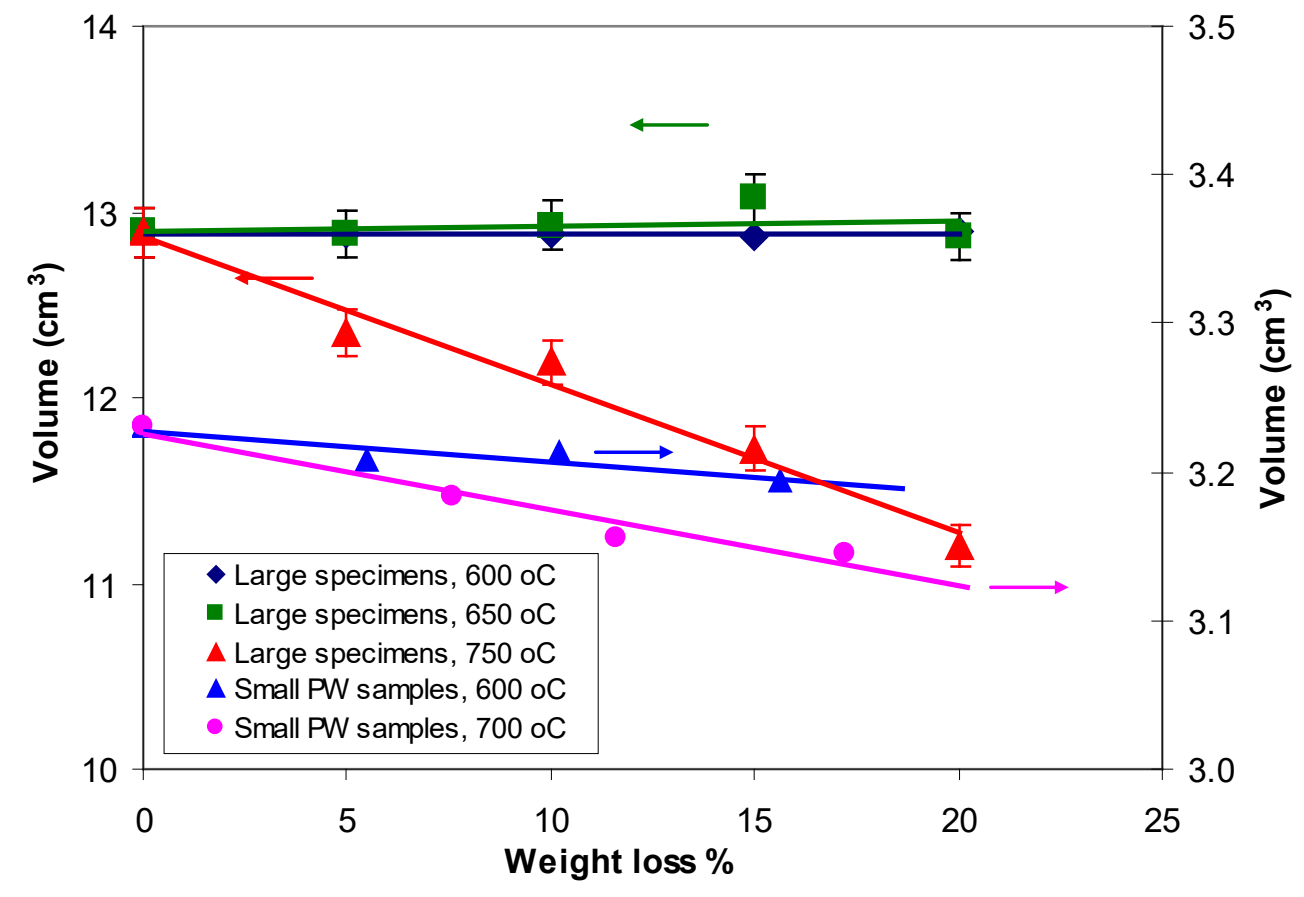

Figure 5 Volume variations of PCEA graphite specimens as a function of weight loss. Data for "large" specimens (25.4 mm diameter) are plotted on the left ordinate axis. Average data for "small" specimens (12.7 $\mathrm{mm}$ diameter) are plotted on the right ordinate axis. 


\subsection{Automated Image Collection using Optical Microscopy}

For examination by optical microscopy the specimens were cut at about $1 / 3$ of the length. The shorter pieces were encased in resin and the surface exposed after cutting was polished using several grades (from gross to fine) of alumina powder.

A Nikon Microphot FXA microscope (Japan) was used for image collection. It is equipped with motorized stage and predictive autofocus control. Digital images were acquired using the Leica Application Suite version 3.1.0 (Leica Microsystems CMS GmbHm Switzerland). All images were collected on the monochromatic 8-bit grey scale, where the brightness of individual pixels ranges between 0 (extreme dark) and 255 (extreme white). Since uniform illumination of the view field is critical for the quality of mosaic images, the microscope was upgraded in 2010 by replacing the illumination source with a pre-centered bright light source connected through optical fiber to the microscope (Nikon Intensilight model C-HGFI). This upgrade improved substantially the quality of photomicrographs.

Optical microscopy makes it possible to analyze the spatial distribution (or depth) of the oxidized layer relative to the exposed surface (external) of graphite specimens. In order to do that, the automated stage control feature of the microscope was used to collect several hundred microphotographs at medium $(200 \mathrm{x})$ or high magnification $(400 \mathrm{x})$. The micrographs were then assembled in a rectangular mosaic image which covered a surface zone extending from one oxidized edge to another, along the diameter. In 2009 , while working with "large" specimens, full images ( $25.5 \mathrm{~mm}$ x $2 \mathrm{~mm}$ ) could be obtained only with medium magnification $(200 \mathrm{x})$ because of size limitations of large mosaic image files.

This year, the "large" specimens were re-analyzed using higher magnification $(400 \mathrm{x})$ in order to better identify the distribution of porosity in the oxidized layer. However, at this magnification the mosaic image could cover only a shorter zone $(12 \mathrm{~mm} \times 1 \mathrm{~mm})$ from the oxidized edge to the center of "large" specimens. For the new series of "small" specimens produced in 2010, the same magnification (400 x) produced a mosaic image extending from one oxidized edge to another across the diameter $(12.7 \mathrm{~mm} \times 1$ $\mathrm{mm}$ ). These new images were analyzed using the procedure described below.

\subsection{Automated Image Analysis Protocol}

The automated image analysis was done using the Image Pro Plus software version 6.3.0.512 (Media Cybernetics Inc.).

The first step of image analysis is spatial calibration, in order to set the measuring units of the system. A graticule was used for calibration, with traces separated by $0.1 \mathrm{~mm}$ and $0.02 \mathrm{~mm}$. This made it possible to work with real distances rather than conventional pixel units. Spatial calibration of mosaic images was done by collecting images of the calibrated ruler in identical conditions as the graphite images.

The next step is to enhance the contrast of each individual image in order to improve the ability of extracting objects. In order to consistently produce a high contrast image with little dynamic range, we used the exponential equalization function of Image-Pro software. This function analyzes the accumulated histogram and redistributes in a way that enhances the contrast of dark images.

Next, the histogram analysis of each image was performed. Histograms illustrate the distribution of shades of grey in a graphical form, showing the frequency of pixel distribution along a scale of brightness intensities ranging from 0 (extreme black) to 255 (extreme white). The surface of optically polished graphite is in general reflective for visible light and appears bright, with correspondingly high pixel values. Porosity preexistent in graphite does not reflect visible light and is visible as grey (and sometimes black) zones. Similarly, the porosity and surface roughness created through oxidation appear as areas with low reflectivity, namely grey zones with lower than average pixel values.

Using histogram analysis and segmentation of images based on objective criteria makes it possible to analyze changes introduced by oxidation. The oxidized layer appears as a darker zone extending a variable length from the edge of oxidized specimens inwards. After analyzing and comparing numerous images, it was found that the modifications introduced by oxidation are essentially in the range of grey tones.[25] These modifications were identified as peaks (or shoulders) on the histogram at pixel values 
below the median level. An example is shown in Figure 6 which compares histograms for PW samples before oxidation and oxidized at three levels of weight loss at 600 and $700{ }^{\circ} \mathrm{C}$. The pixel intensity intervals used for segmentation of the images (in this example) are between the purple and green marks shown on each histogram in Fig. 6.

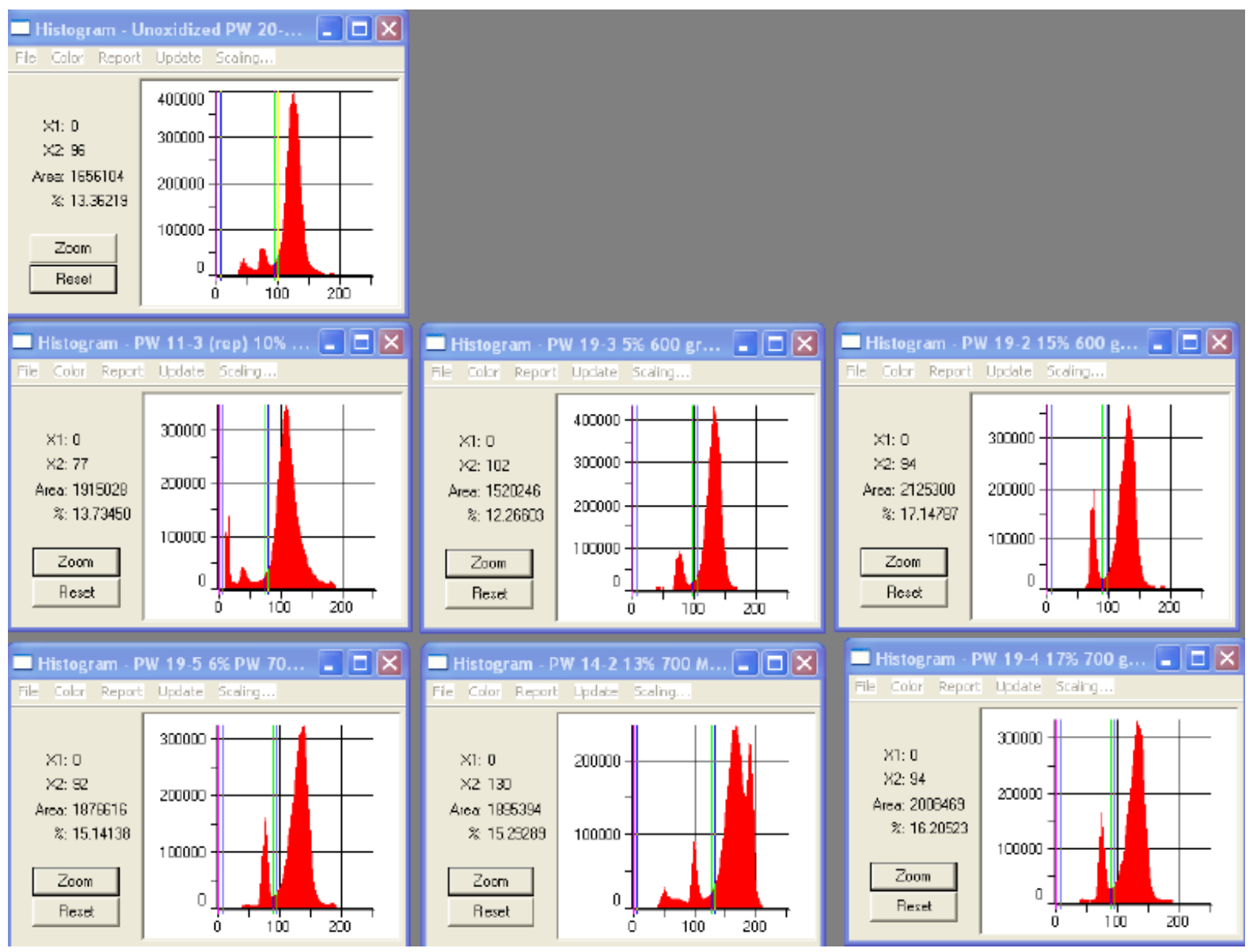

Figure 6 Example of histogram of pixel intensities for a specimen (PW 20-3) un-oxidized (top row); specimens oxidized at $600{ }^{\circ} \mathrm{C}$ (PW 19-3 at 5\% weight loss; PW 11-3 at 10\% weight loss; PW 19-2 at 15\% weight loss) in middle row; and for specimens oxidized at $700{ }^{\circ} \mathrm{C}$ (PW 19-5 at 6\% weight loss; PW 14-2 at 13\% weight loss; PW 19-4 at $17 \%$ weight loss) in the bottom row. All specimens were of "small" sizes $(12.7 \mathrm{~mm}$ diameter). The threshold of pixel intensities selected for further analysis is shown on each picture.

After image segmentation based on pixel intensity and identification of objects, next step is counting of selecting objects, measuring their properties (area in this case) and classifying them based on properties. Figure 7 shows an example of complete sequence of automated image analysis for un-oxidized PW 10-3 specimen of $12.7 \mathrm{~mm}$ diameter. The mosaic image $(12.970 \mathrm{~mm} \times 1.067 \mathrm{~mm})$ was assembled from 576 individual microphotographs, distributed in 8 rows of 72 fields each. The size of each field was $180 \mu \mathrm{m}$ x $135 \mu \mathrm{m}$. Magnification was $400 \mathrm{x}$. The individual pixel lateral size was $3.156 \mu \mathrm{m}$ which results in $9.96 \mu \mathrm{m}^{2}$ for the area of the smallest measurable objects. The pores were identified based on the pixel 
intensity histogram. The range selected was between 0 and 96, see Fig. 6 (top row). A number of 8926 objects were found; they were measured, counted and classified based on size (pore area). Two examples of classification are shown, where the objects (pores) were classified in either two bins or ten bins, based on size.

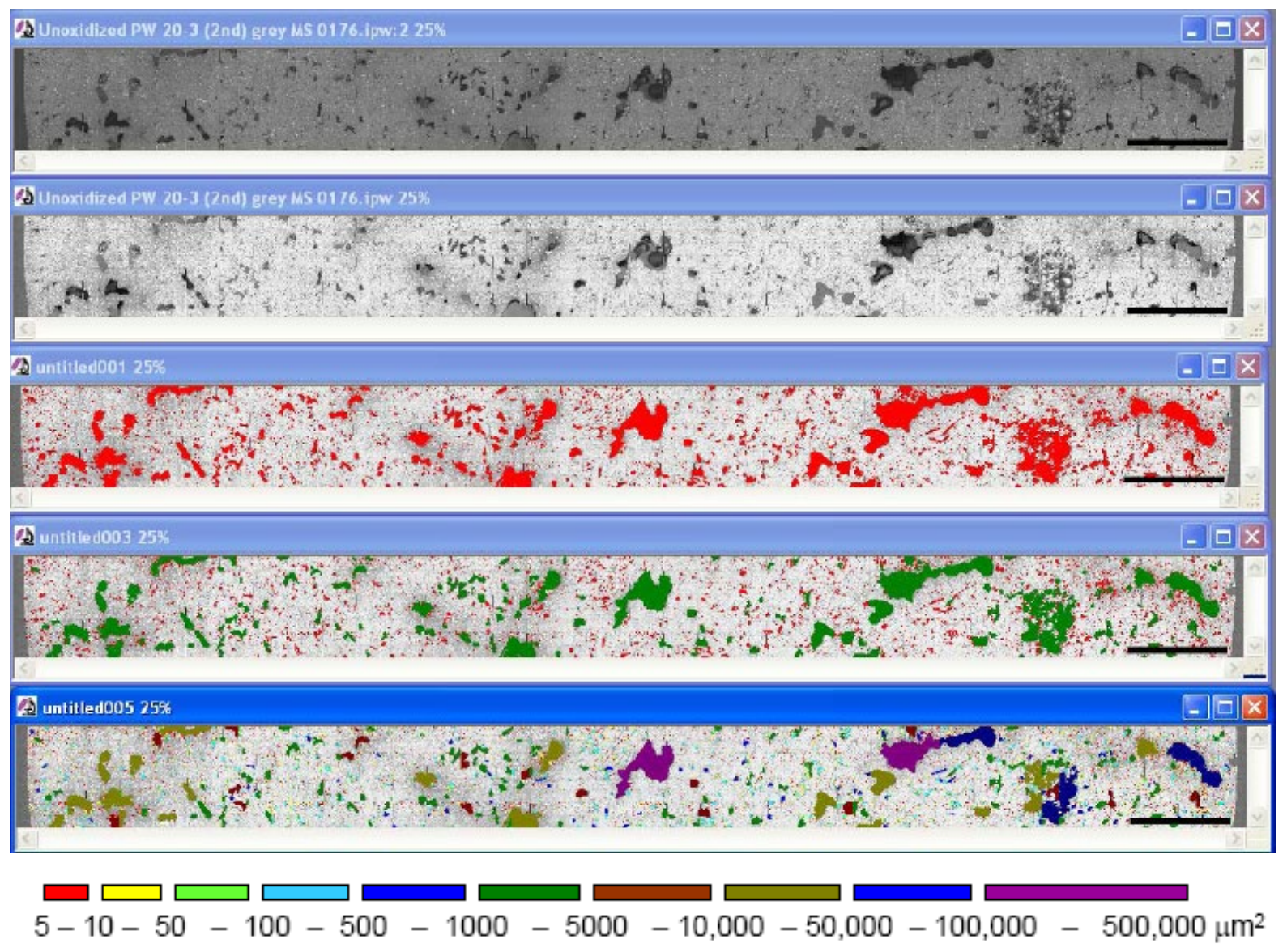

Figure 7 Example of an automated image analysis sequence applied to unoxidized specimen PW 10-3 in series of "small" graphite samples (12.7 mm diameter). From top to bottom, the screen snapshots represent (a) original image; (b) contrast-equalized image using an exponential function; (c) result of segmentation using the range of pixel intensities between 0 and 96 identified on the histogram in Fig. 6, top row; (d) object classification based on size, using two classes (red: $5-1000 \mu \mathrm{m}^{2}$ and green: $1000-500,000 \mu \mathrm{m}^{2}$ ); and (e) object classification based on size, using ten classes (color scale shown). The scale bar in all images is $1 \mathrm{~mm}$. Images have been reduced to $25 \%$ of original size for fitting on page.

Pore size distribution of un-oxidized PCEA graphite is also available from mercury porosimetry measurements (Figure 8).[29] This graphite has a bi-modal pore distribution, with about $50-55 \%$ of total pore volume in pores with diameter smaller than $0.1 \mu \mathrm{m}$, and about $45-50 \%$ of total pore volume in pores with diameter between $\sim 3.5$ and $120 \mu \mathrm{m}$. When normalized to sample volume, the largest pores $(5-120$ $\mu \mathrm{m})$ represent about $6 \%$ of total material's volume. Obviously, only these pores are visible in optical 
microscopy. Their projection on a flat surface is between 9.6 and $11,300 \mu \mathrm{m}^{2}$ if their cross-section shape is round, or 12.2 and $14,400 \mu \mathrm{m}^{2}$ if their cross-section is square. Larger pores are also present. One example is shown in the bottom row of Fig. 7 where the two pores colored purple have areas of 108,000 and $122,000 \mu \mathrm{m}^{2}$ which correspond to maximum Feret dimensions between 600 and $765 \mu \mathrm{m}$.
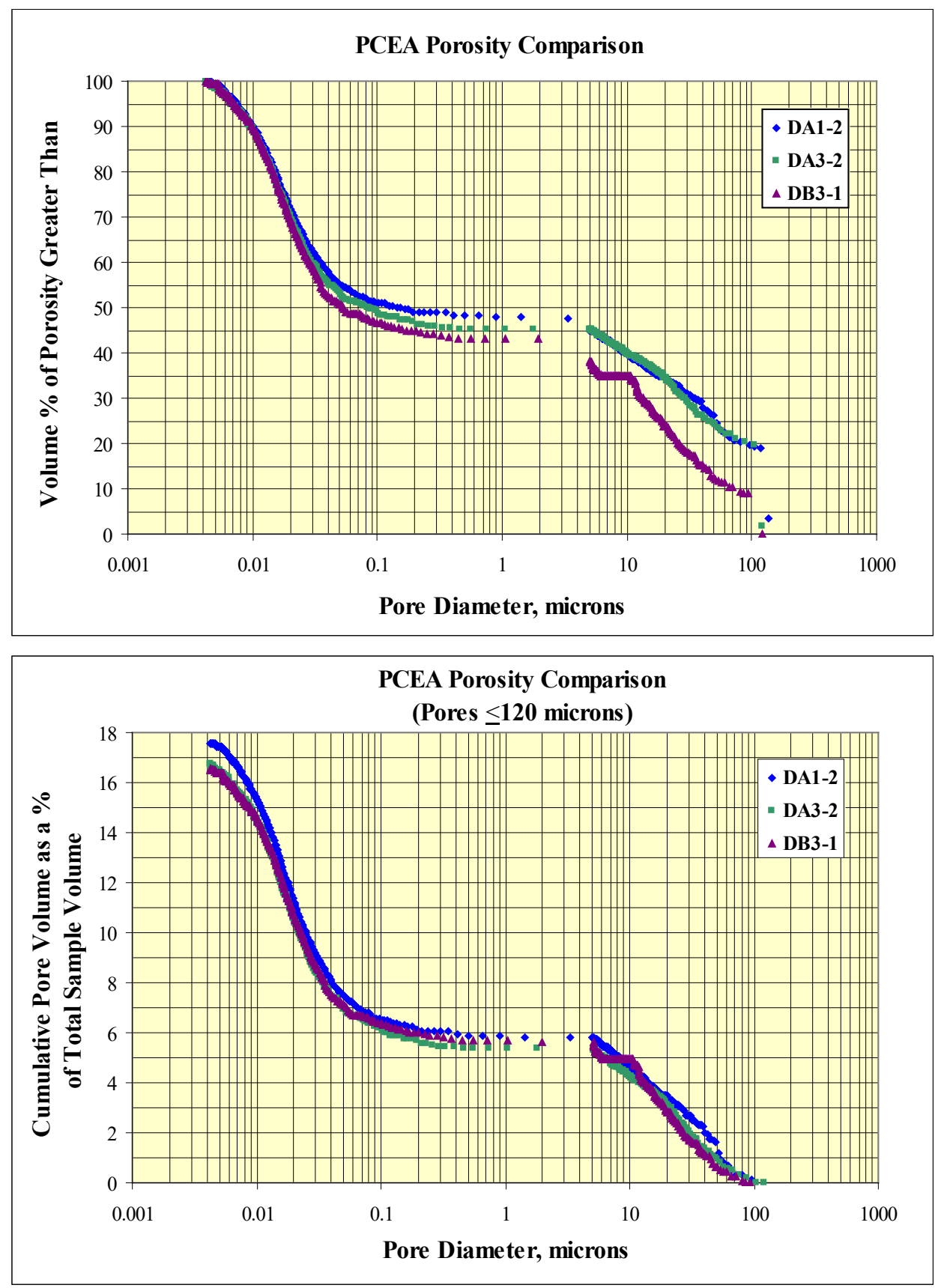

Figure 8 Distribution of open pore volume in un-oxidized PCEA graphite measured by mercury intrusion porosimetry. Data are normalized by total pore volume (top panel) and by total sample volume (bottom panel). Redrawn from ref. [29]

This comparison shows that there is a good agreement between the pore sizes measured by optical microscopy and by mercury intrusion porosimetry. 


\subsection{Image Analysis Results}

\subsubsection{Distribution and thickness of oxidized layers}

Automated image analysis of the new series of photomicrographs collected for "large" PCEA graphite specimens $(25.4 \mathrm{~mm}$ diameter) provided better insight in the distribution and evolution of porosity in the oxidized layer. These samples (see Table 4 for identification) have been obtained and analyzed first time in 2009. [25] The results obtained through image analysis of low magnification micrographs $(200 \mathrm{x})$ have shown that the oxidized layer has variable thickness, depending on the temperature of oxidation (in air): about $6-7 \mathrm{~mm}$ at $600{ }^{\circ} \mathrm{C}$; about $3-4 \mathrm{~mm}$ at $650{ }^{\circ} \mathrm{C}$; and less than $1 \mathrm{~mm}$ at $750^{\circ} \mathrm{C}$. However, it was not clear what the mechanism of oxidation front advancement was as a function of weight loss, and how is porosity created by oxidation.

A new series of images of these "large" specimens were collected at higher magnification $(400 \mathrm{x})$. They were analyzed following the protocol introduced above. The objects have been classified in 10 bins based on size, with sizes between 5 and 500,000 $\mu \mathrm{m}$. Mosaic images shown in Figures $9-11$ show the sequence of development of the oxidation layer with the increase of weight loss at three temperatures, 600,650 , and $750{ }^{\circ} \mathrm{C}$. The size of mosaic images is $12 \mathrm{~mm} \times 1 \mathrm{~mm}$.

From Figs. 9-11 it is visually apparent how the thickness of the oxidized layer varies with the oxidation temperatures. This variation is quantified in Figure 12, which shows area profiles of pixel intensities for all oxidizes samples in Figs. 9-11 (see also Table 3 for identification of specimens). Area profiles are plots of pixel intensities distributed by distance (in $\mu \mathrm{m}$ ) from the exposed surface. Low values of pixel intensities show grey or dark objects (pores). It is important to observe that all area profiles at a selected temperature have the same aspect; they do not vary with the level of burn-off. The slope become higher as the temperature increases, showing that the thickness of the oxidized layer diminishes. These observations shed a light on the mechanism of pore development, which will be discussed next. 
Q

$-\sqrt{\mathrm{x}}$
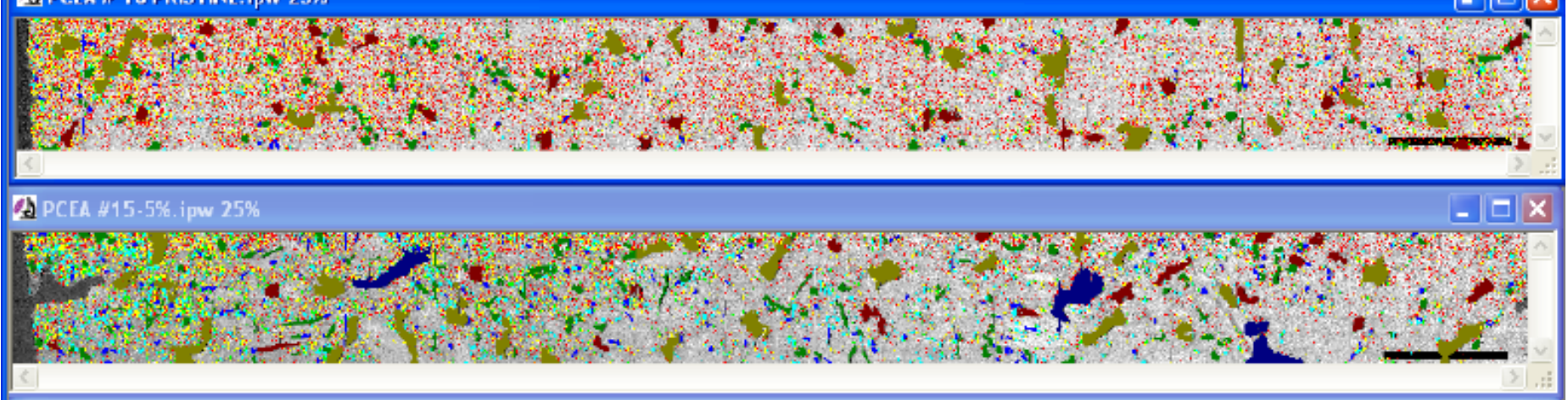

2. PCEA H14-10\%. Ipw 25\%

$-\square \times$
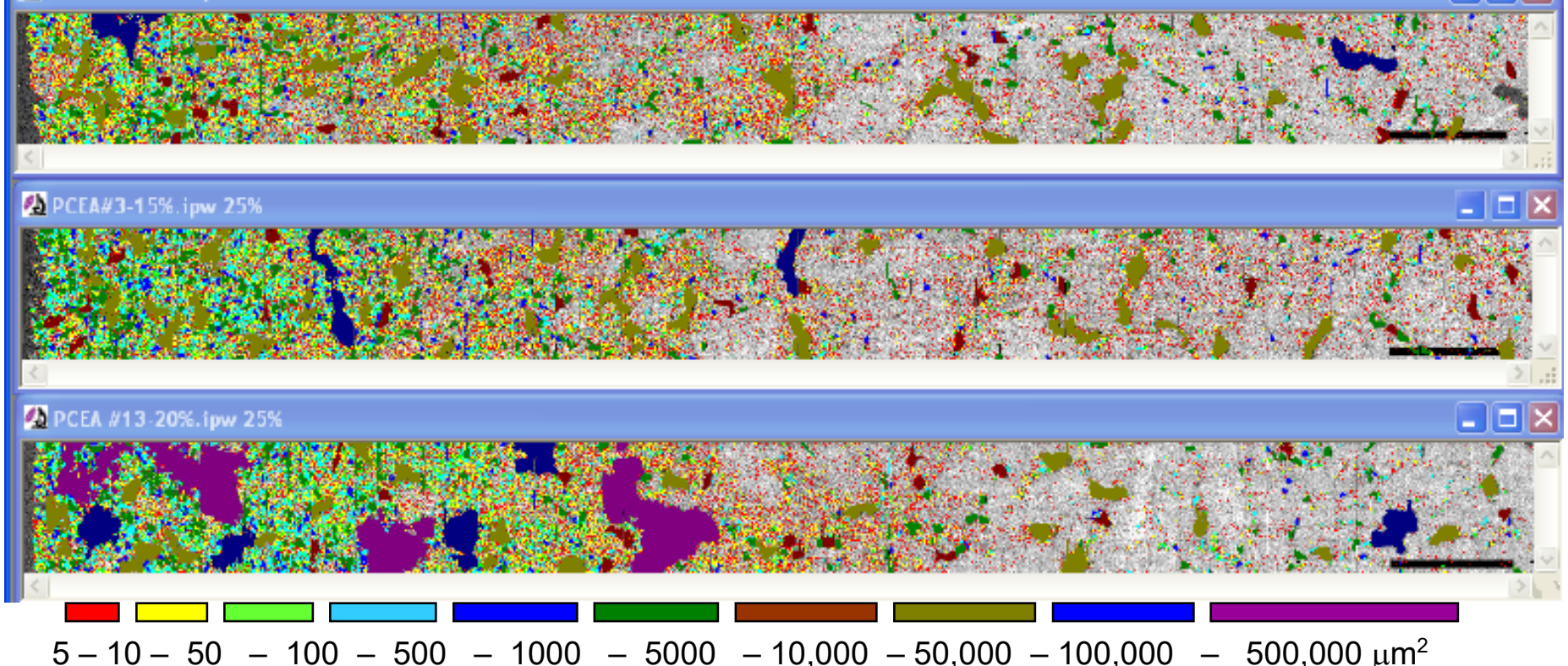

Figure 9 Development of oxidized layer in "large" PCEA graphite specimens at $600{ }^{\circ} \mathrm{C}$. From top to bottom: pristine specimen (\#18), and specimens oxidized at $5 \%$ weight loss (\#15), $10 \%$ weight loss (\#14), $15 \%$ weight loss (\#3), and $20 \%$ weight loss (\#13). See Table 3 for specimen identification. Magnification $400 \mathrm{x}$. Scale bar is $1 \mathrm{~mm}$. 


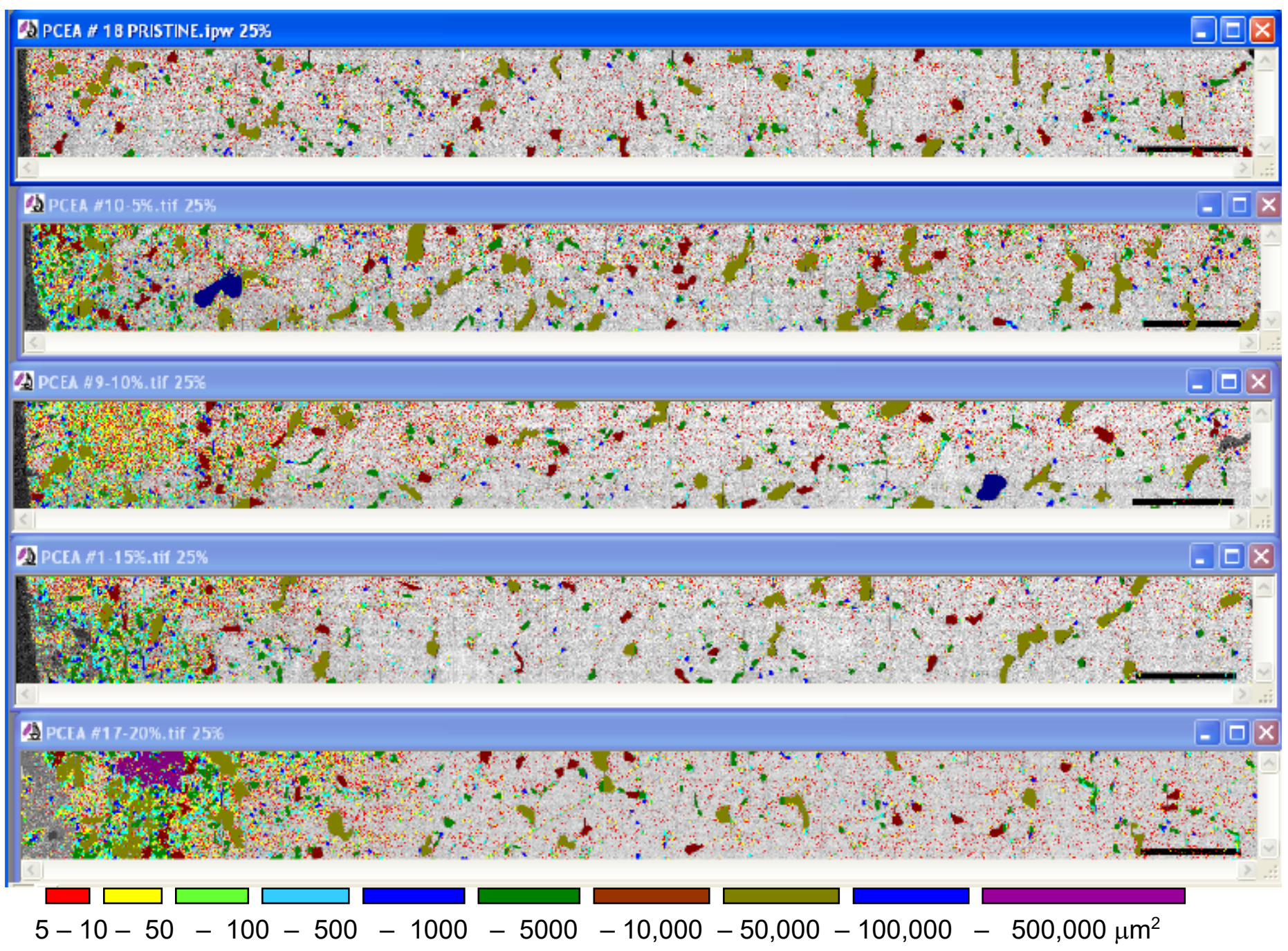

Figure 10 Development of oxidized layer in "large" PCEA graphite specimens at $650{ }^{\circ} \mathrm{C}$. From top to bottom: pristine specimen (\#18), and specimens oxidized at $5 \%$ weight loss (\#10), $10 \%$ weight loss (\#9), $15 \%$ weight loss (\#1), and $20 \%$ weight loss (\#17). See Table 3 for specimen identification. Magnification $400 \mathrm{x}$. Scale bar is $1 \mathrm{~mm}$. 


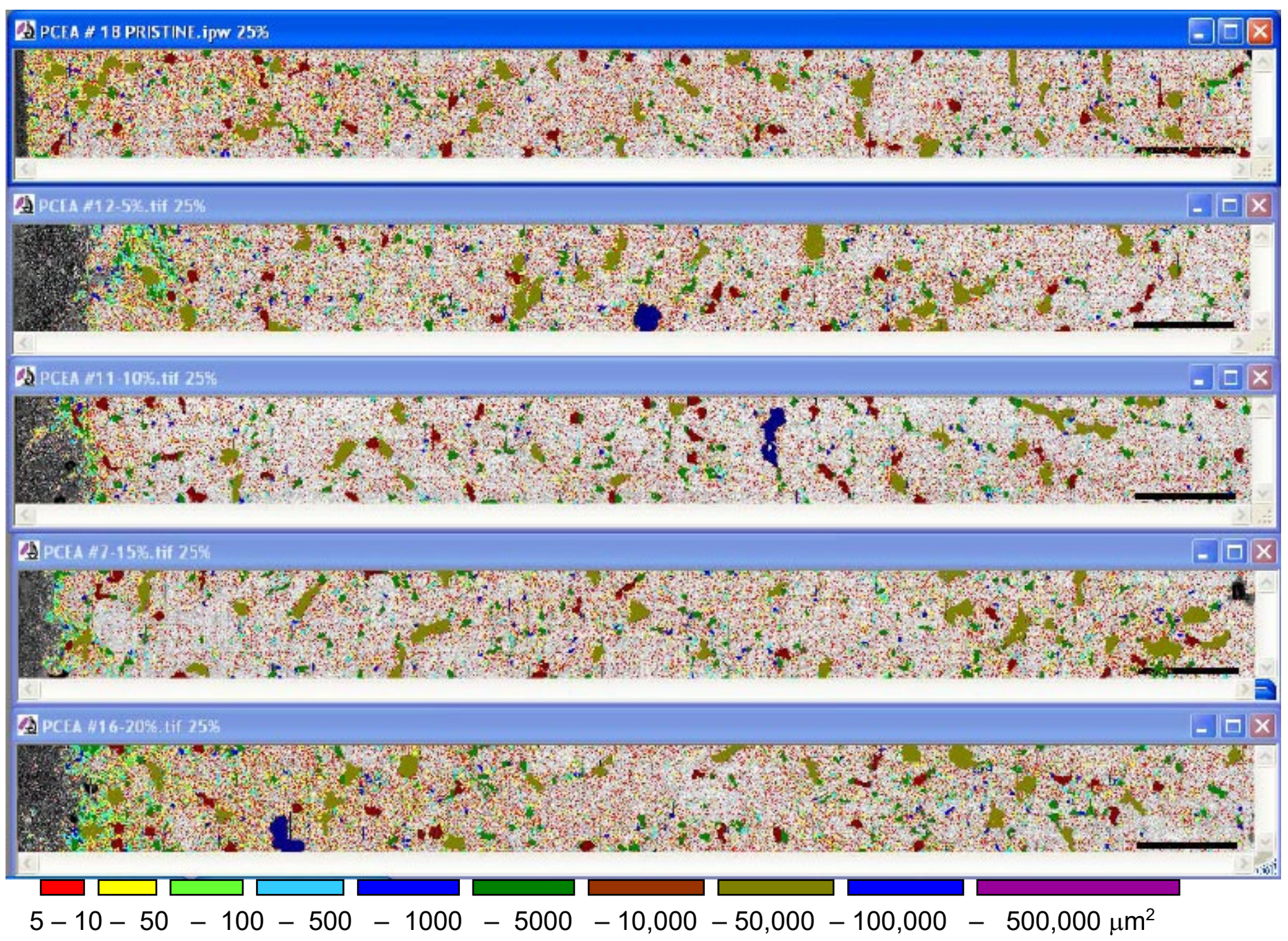

Figure 11 Development of oxidized layer in "large" PCEA graphite specimens at $750{ }^{\circ} \mathrm{C}$. From top to bottom: pristine specimen (\#18), and specimens oxidized at $5 \%$ weight loss (\#12), $10 \%$ weight loss (\#11), $15 \%$ weight loss (\#7), and $20 \%$ weight loss (\#16). See Table 3 for specimen identification. Magnification $400 \mathrm{x}$. Scale bar is $1 \mathrm{~mm}$. 
$5 \%$ at $600 \mathrm{C}(\# 15)$

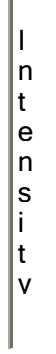

$\left[\begin{array}{l}\text { I } \\ n \\ t \\ e \\ n \\ s \\ i \\ t \\ v\end{array}\right.$

\begin{tabular}{|l} 
I \\
n \\
t \\
e \\
n \\
s \\
i \\
t \\
v
\end{tabular}

Figure 12

Distance (um)
$10 \%$ at $600 \mathrm{C}(\# 14)$

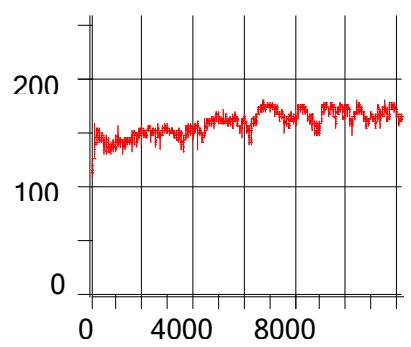

$10 \%$ at $650 \mathrm{C}(\# 9)$

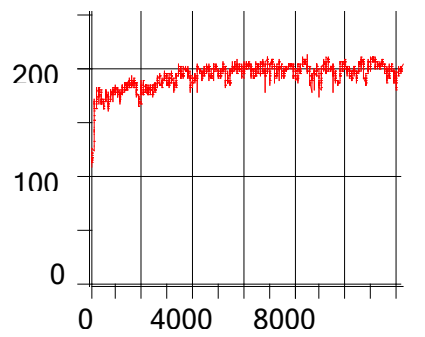

$10 \%$ at $750 \mathrm{C}(\# 11)$

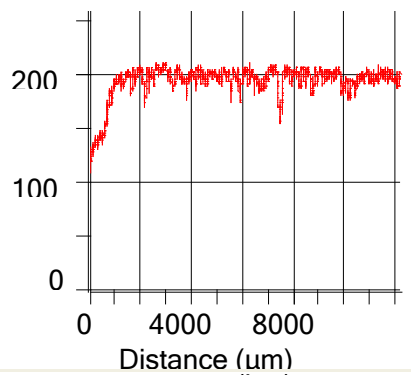

$15 \%$ at $600 \mathrm{C}(\# 3)$

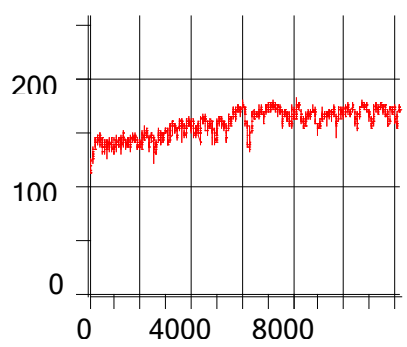

$15 \%$ at $650 \mathrm{C}(\# 1)$

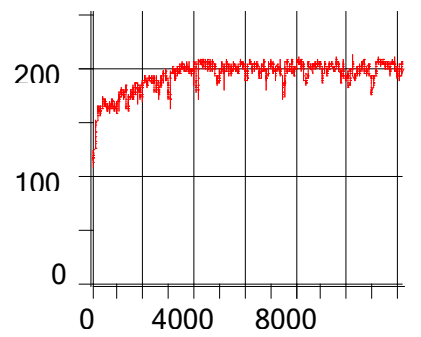

$15 \%$ at $750 \mathrm{C}(\# 7)$

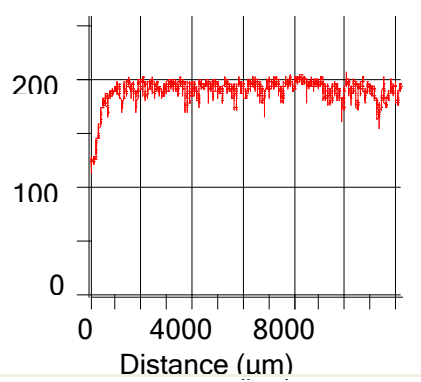

$20 \%$ at $600 \mathrm{C}(\# 13)$

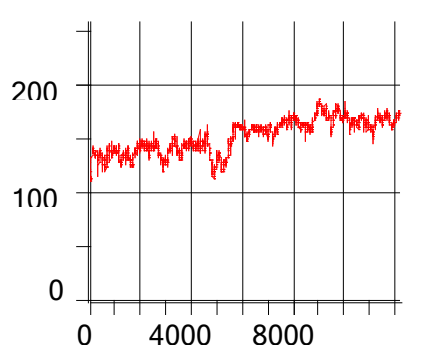

$20 \%$ at $650 \mathrm{C}(\# 17)$

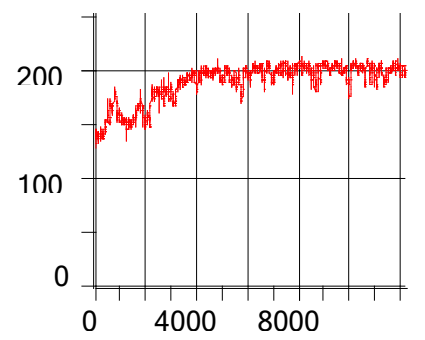

$20 \%$ at $750 \mathrm{C}$ (\#16)

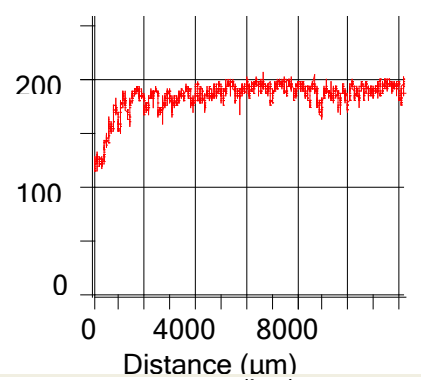

Area profile histograms through the oxidized layer in "large" PCEA specimens, showing radial distribution of the oxidized layer. Vertical axis represents pixel intensities on the 0-255 scale. Horizontal axis is distance in $\mu \mathrm{m}$ from surface. Small pixel values 
near the edge of the specimen are identified with higher density of pores in the oxidized layer. See Table 3 for specimen identification. 


\subsubsection{Mechanism of pore development}

In order to understand the mechanism of pore development, we focused analysis on two areas of interests in the oxidized layer of specimens with variable weight loss obtained at $600{ }^{\circ} \mathrm{C}$ :

(1) between 1 and $2 \mathrm{~mm}$ from the exposed surface, in the middle of the oxidation layer

(2) between 5 and $6 \mathrm{~mm}$ from the exposed surface, at the transition between the oxidized layer and the core, apparently not touched by oxidation at $600{ }^{\circ} \mathrm{C}$.

Figure 13 shows images of $1 \mathrm{~mm} \times 1 \mathrm{~mm}$ areas of selected zones. The top row in this figure shows that in the middle of the oxidation (2-3 mm from surface) the increase of weight loss is accompanied by increase in pore sizes, merging of smaller pores into larger pores, and eventually collapse of the pore structure and development of large size voids. The bottom row shows changes occurring at 5-6 $\mathrm{mm}$ from surface, at the limit between the oxidized layer and the core. Here, the progress of weight loss generates higher densities of small pores, which develop preferentially in the binder phase, while graphite grains are not visibly affected. The density of newly developed narrow pores is larger in the areas surrounding large pores that pre-exist in graphite. This observation suggests that larger pores serve as transport channels for oxidant, and newly developed narrow pores radiate from large pores and spread to the binder phase. Because of the graphitization degree which depends on the process temperatures, the binder phase in graphite has a lower oxidation resistance. [30, 31].

Figure 14 shows more details of the oxidized layer in the transition zone (5-6 $\mathrm{mm}$ from surface). The top row images in Fig. 14 were collected at $400 \mathrm{x}$ magnification and correspond to the same areas as in the bottom row images of Fig. 13. The pores (objects) were auto-classified in three classes based on size, using the auto-classification routine of Image Pro Plus. In this classification the binder phase appears as a mosaic of small crystallites, colored in shades of blue and pink. The bottom row of micrographs in Fig. 14 show more details at $1000 \mathrm{x}$ magnification. Surface imperfections caused by polishing traces are more visible at this magnification. However, preferential development of the new pores in the binder and their high aspect ratio is very visible. 
Oxidation at $600^{\circ} \mathrm{C}: 2-3 \mathrm{~mm}$ from edge
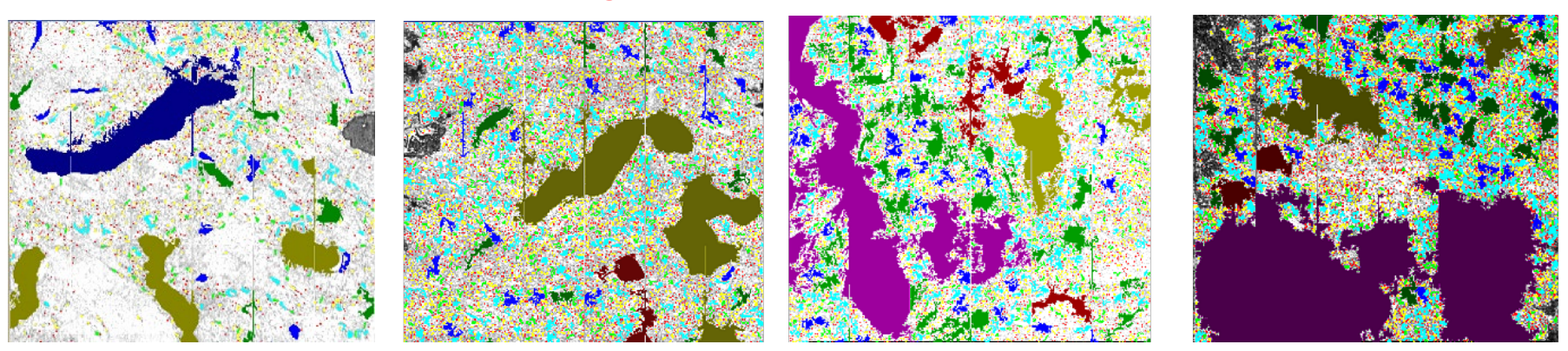

Oxidation at $600{ }^{\circ} \mathrm{C}: 5-6 \mathrm{~mm}$ from edge
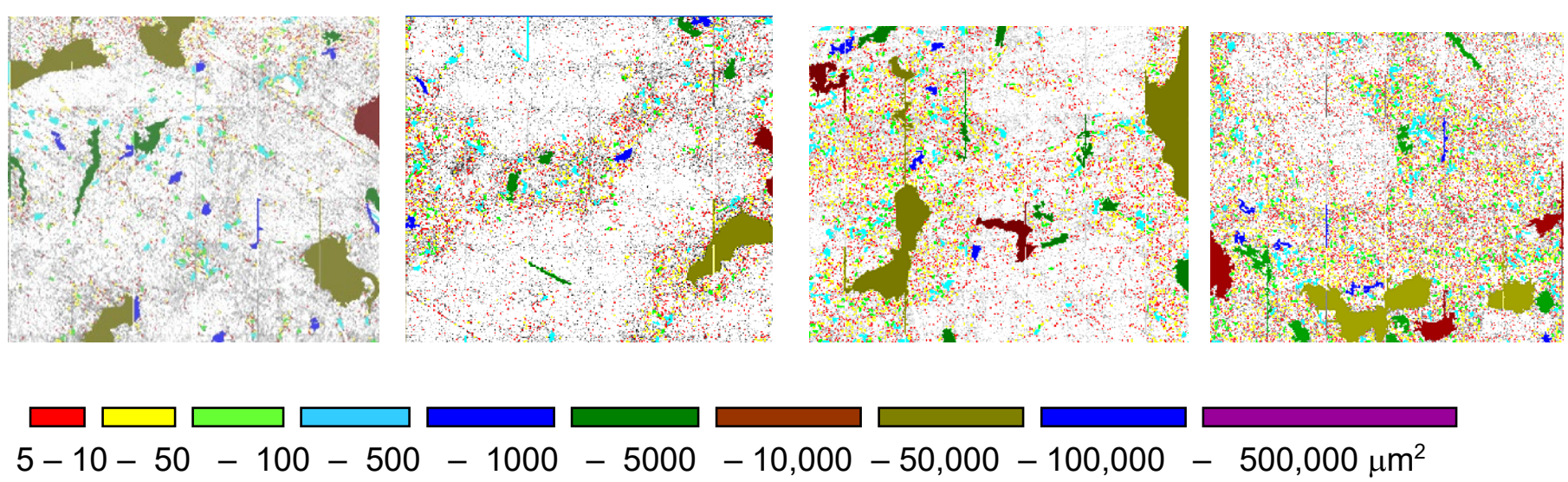

Figure 13

Evolution of oxidized layer structure with the level of weight loss at $600{ }^{\circ} \mathrm{C}$. The two areas of interest shown correspond to 2-3 $\mathrm{mm}$ and 5-6 $\mathrm{mm}$ from the edge of oxidized graphite specimens. The size of each image is $1 \mathrm{~mm}^{2}$. Magnification $400 \mathrm{x}$. 
$5 \%$ at $600 \mathrm{C}$

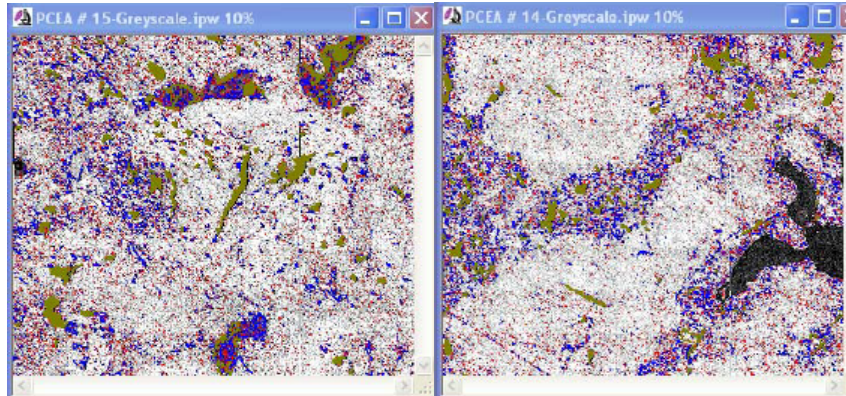

$5 \%$ at $600 \mathrm{C}$

$10 \%$ at $600 \mathrm{C}$
$15 \%$ at $600 \mathrm{C}$
$20 \%$ at $600 \mathrm{C}$

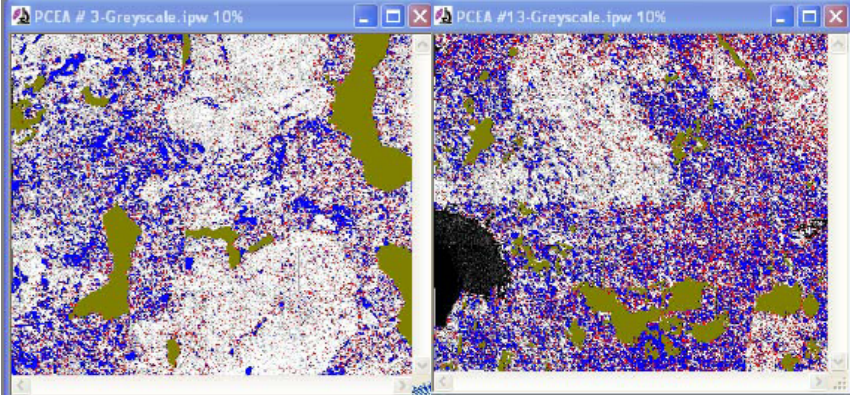

Image size: $1000 \mu \mathrm{m} \times 1000$

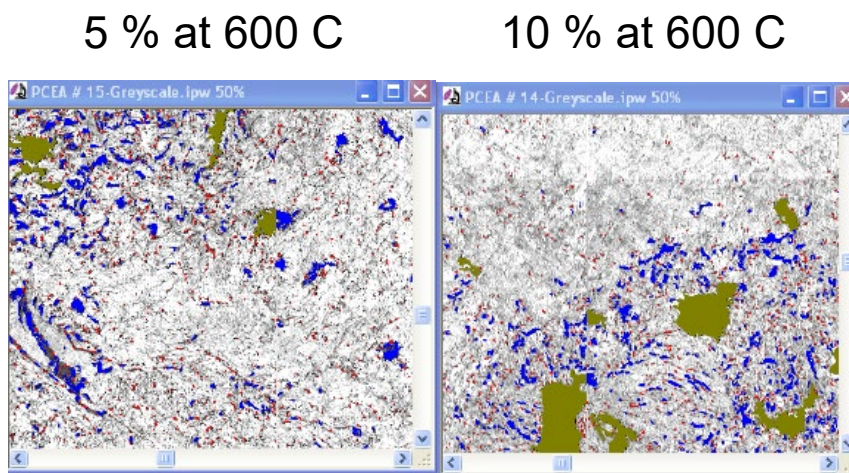

$15 \%$ at $600 \mathrm{C}$

$20 \%$ at $600 \mathrm{C}$

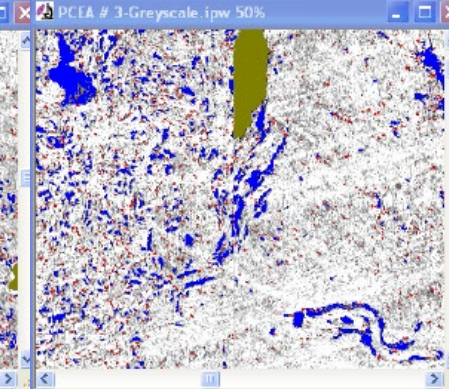

Image size: $250 \mu \mathrm{m} \times 250$

Figure 14

Another view of images showing the structure of oxidized layer at $5-6 \mathrm{~mm}$ from surface for specimens oxidized at $600{ }^{\circ} \mathrm{C}$ in air. Top: images at $400 \mathrm{x}$ magnification in the same areas as in bottom row images of Fig. 13, but using auto-classification routine of Image Pro Plus software. Bottom: detail images at $1000 \mathrm{x}$ magnification in the same areas as of top row. All images show that oxidation causes primary development of narrow pores in the binder phase. 
Observations by optical microscopy lead to the following conclusions on the mechanism of pore growth during air oxidation:

- The primary channel for transport of oxidant is the network of pores pre-existent in graphite. These pores, identified by mercury intrusion porosimetry, are interconnected. From these pores where the oxidant has facile transport, oxidation spreads out to the neighboring material.

- The depth of oxidized layer depends primarily on temperature, but seems to be independent on the degree of oxidation. The penetration depth of the oxidant is balanced by two opposing processes: transport through large pores and consumption through attack of material in neighboring pores.

- The binder is oxidized first. Oxidation causes development of new, high aspect ratio pores in the binder phase in volumes surrounding pre-existent large pores, which weakens the binder and thus the graphite.

- With the progress of isothermal oxidation, consumption of graphite occurs mainly through the growth, coalescence, and finally collapse of pores in the oxidized layer. At low and moderate temperatures the inwards advancement of the oxidized layer does not occur with significant rate. Only after the material has been consumed at some critical extent, the oxidized layer advances inwards if isothermal conditions continue.

- At higher temperatures the penetration of oxidant is limited at all times. Oxidation causes rapid and substantial corrosion at the exposed surface.

- The mechanisms of pore transport and chemical oxidation compete in the temperature range investigated where oxidation rates are conveniently measurable (between about 550 and 750 ${ }^{\circ} \mathrm{C}$ for oxidation in air). Because the oxidant can use the network of large pores for fast transport inwards from surface, material consumption in the oxidized layer occurs with apparent rates not much different from actual chemical oxidation rates (provided there is large enough supply of oxidant).

- When sufficient supply of oxidant is allowed from the gas phase, oxidation rates follow (more or less) an Arrhenius-type dependence with temperature. This is apparently in contradiction with frequent observations of non-uniform oxidation profiles, as reported by Contescu et al. [6] A possible explanation, previously offered by Eto and Growcock [16], is the coexistence of two systems of pores in graphite (large, for fast transport, and small, where chemical oxidation actually takes place) - see also Fig. 8.

\subsubsection{Effect of specimen size on oxidation layer penetration}

One specimen of each oxidized lot of "small" PCEA specimens machined in 2010 (12.7 mm diameter) has been randomly selected for optical microscopy analysis. The procedure described above was followed. High magnification images were taken of a strip $12.9 \mathrm{~mm} \times 1 \mathrm{~mm}$ in size across the center of specimens. Figures 15 and 16 show pore classification in two bins defined as pores smaller or larger than $1000 \mu \mathrm{m}^{2}$. Figure 17 shows area profiles of pixel intensities for the same samples. Area profiles were calculated by averaging all pixel intensities in mosaic images as a function of position from one edge to another. 

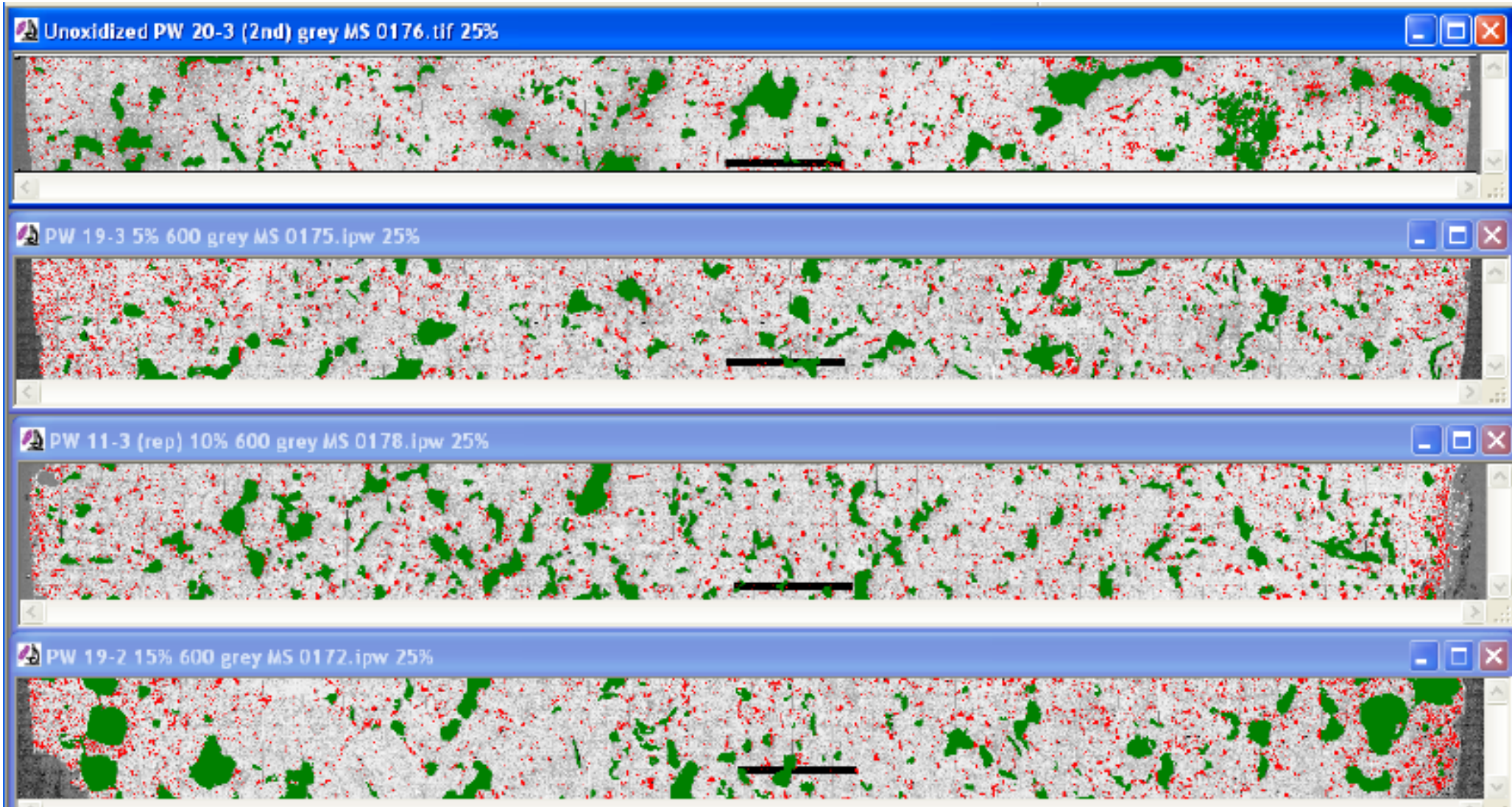

Figure 15

Pore structure of "small" PCEA specimens $(12.7 \mathrm{~mm}$ diameter $)$ oriented "with grain" after oxidation in air at $600{ }^{\circ} \mathrm{C}$. From top to bottom: PW 20-3 (un-oxidized); PW 19-3 (5\% weight loss); PW 11-3 (10\% weight loss); PW 19-2 (15\% weight loss). Scale bar is $1 \mathrm{~mm}$. Color code: red $=$ pores $<1000 \mu \mathrm{m}^{2}$; green $=$ pores $<1000 \mu \mathrm{m}^{2}$. 


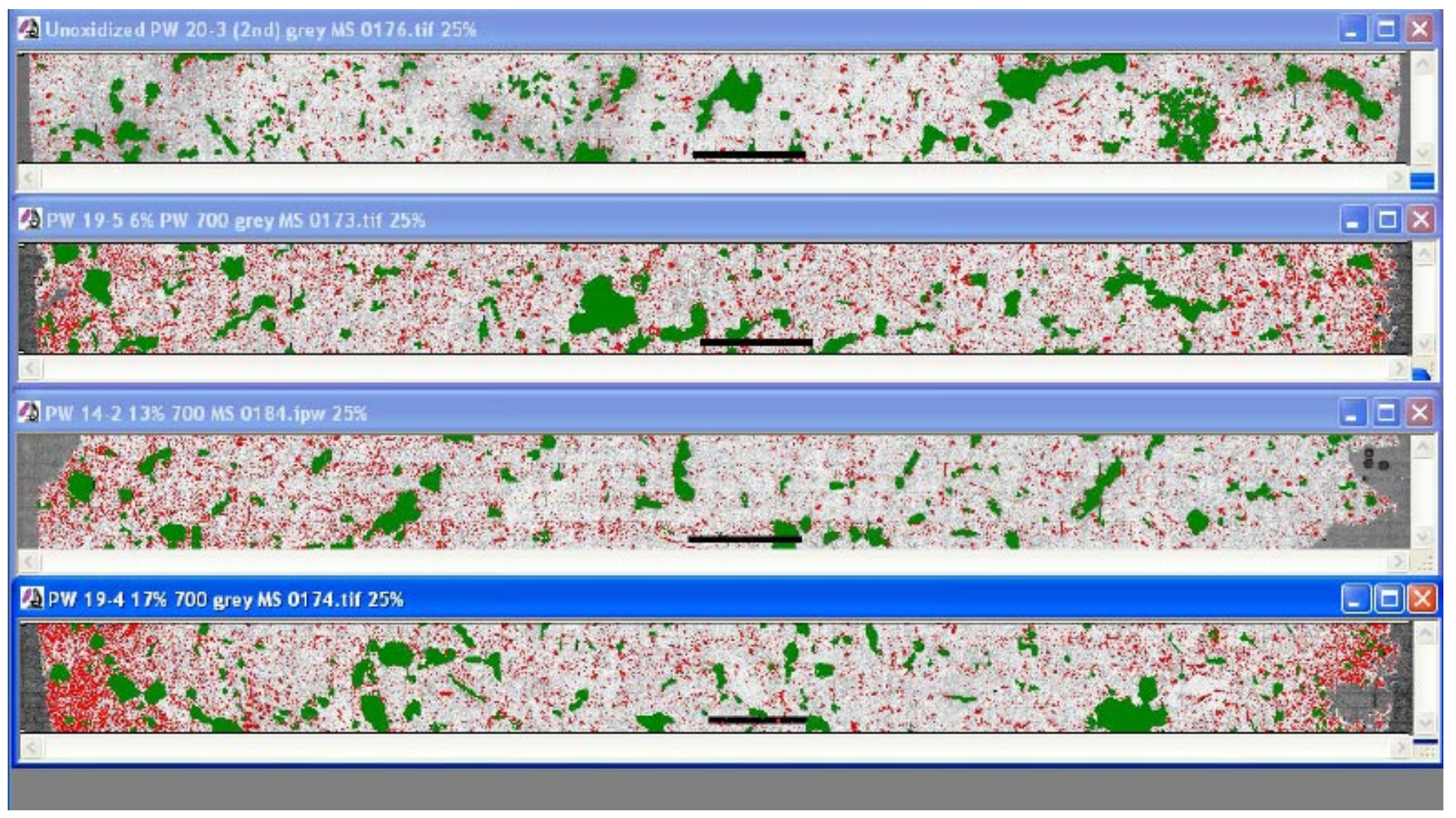

Figure 16

Pore structure of "small" PCEA specimens $\left(12.7 \mathrm{~mm}\right.$ diameter) oriented "with grain" after oxidation in air at $700{ }^{\circ} \mathrm{C}$. From top to bottom: PW 20-3 (un-oxidized); PW 19-5 (6\% weight loss); PW 14-2 (10\% weight loss); PW 19-4 (17 \% weight loss). Scale bar is $1 \mathrm{~mm}$. Color code: red $=$ pores $<1000 \mu \mathrm{m}^{2}$; green $=$ pores $<1000 \mu \mathrm{m}^{2}$. 

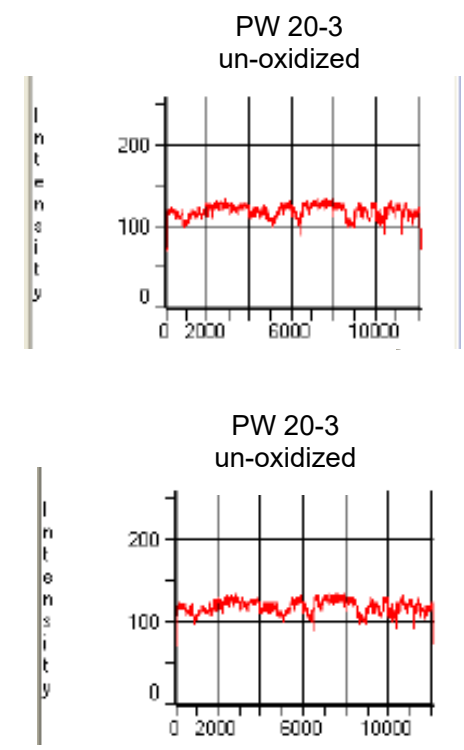

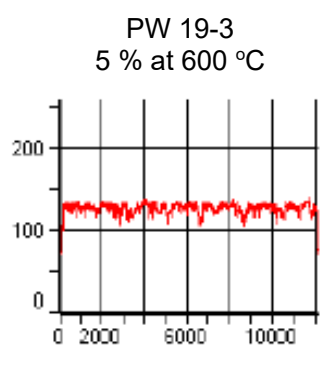

PW $19-5$ $6 \%$ at $700^{\circ} \mathrm{C}$

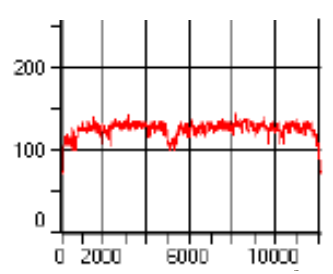

PW 11-3

$10 \%$ at $600{ }^{\circ} \mathrm{C}$

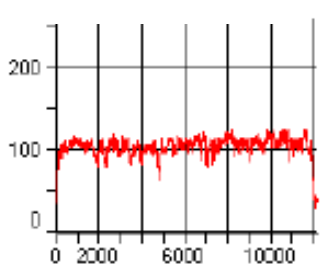

PW 14-2 $13 \%$ at $700{ }^{\circ} \mathrm{C}$

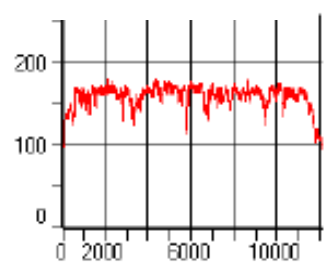

PW 19-2

$15 \%$ at $600{ }^{\circ} \mathrm{C}$

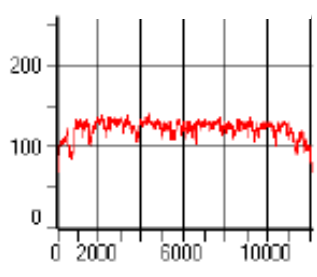

PW 19-4 $17 \%$ at $700{ }^{\circ} \mathrm{C}$

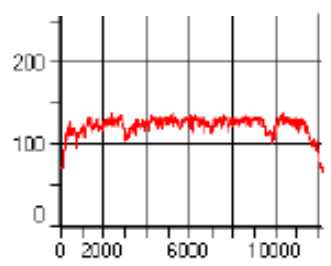

Figure 17

Area profile histograms across "small” PCEA specimens (12.7 mm diameter) showing spatial distribution of the oxidation layer. Vertical axis represents pixel intensities on the 0-255 scale. Horizontal axis is distance in $\mu \mathrm{m}$ from surface. Small pixel intensities near the edges of the specimen are identified with higher density of pores produced by oxidation. See Table 3 for specimen identification

The histograms and pore classification images show that distribution of porosity is more uniform in "small" specimens oxidized at $600{ }^{\circ} \mathrm{C}$ (with possible deviation of sample PW 19-2 oxidized at $15 \%$ weight loss) than in specimens oxidized at $700{ }^{\circ} \mathrm{C}$. At the first glance, this confirms the finding that the thickness of oxidized layer in PCEA graphite is about 6-7 mm at $600{ }^{\circ} \mathrm{C}$ in air. The specimens with 12.7 $\mathrm{mm}$ diameter are fully penetrated by the oxidant at 5 and $10 \%$ weight loss. However, the question remains why specimen PW 19-2 oxidized at $600{ }^{\circ} \mathrm{C}$ to $15 \%$ weight loss exhibits non-uniform profile near the surface. This is shown by the increased density of pores $<1000 \mu \mathrm{m}^{2}$ in Fig. 15 and by the drop of area histogram profile near the surface for sample PW 19-2 in Fig. 17.

A quick calculation shows that this is an expected result. The initial volume of "large" specimens (25.4 mm diameter) is $12.9 \mathrm{~cm}^{3}$. Based on optical inspection the penetration thickness of the oxidized layer is estimated at $6 \mathrm{~mm}$ at $600{ }^{\circ} \mathrm{C}$. However, it is evident that the distribution of density is not uniform in this layer. Based on microscopy images it appears that the maximum extent of pore development occurs in a narrower zone next to the surface. From Fig. 9 one can roughly estimate that the thickness of acute oxidation is about $1 \mathrm{~mm}$ from the exposed surface. Replacing (for this calculation) the shallow density profile in the oxidized layer with a steep profile at about $1 \mathrm{~mm}$ from the surface, one calculates that the annular volume affected by oxidation would be $2.8 \mathrm{~cm}^{3}$. This volume is comparable to the total volume of "small" specimens $\left(3.2 \mathrm{~cm}^{3}\right)$. The conclusion is that some surface corrosion of "small" specimens is to be expected at $600{ }^{\circ} \mathrm{C}$ as the weight loss increases (e.g. $15 \%$ ) even though that was not observed with "large" specimens at the same temperature.

Surface corrosion is more prominent in "small" specimens oxidized at $700{ }^{\circ} \mathrm{C}$ and starts at lower weight loss $(5 \%)$.

The results reported above are in line with previous reports [6,32,33] and model calculations [34] on graphite oxidation. 


\section{EFFECT OF OXIDATION ON COMPRESSIVE STRENGTH LOSS}

\subsection{Compressive Strength Testing Protocol}

The "small" PCEA specimens machined in 2010 (12.7 mm diameter and $25.4 \mathrm{~mm}$ length) were tested according to ASTM C-695, "Standard test method for compressive strength of carbon and graphite" [27] as modified by ASTM C-781, Standard practice for testing of graphite and boronated graphite components for high temperature nuclear reactors" [35] (Annex A5, "Modification to test method C 695"). Figure 18 shows the Instron apparatus used for compressive strength testing.
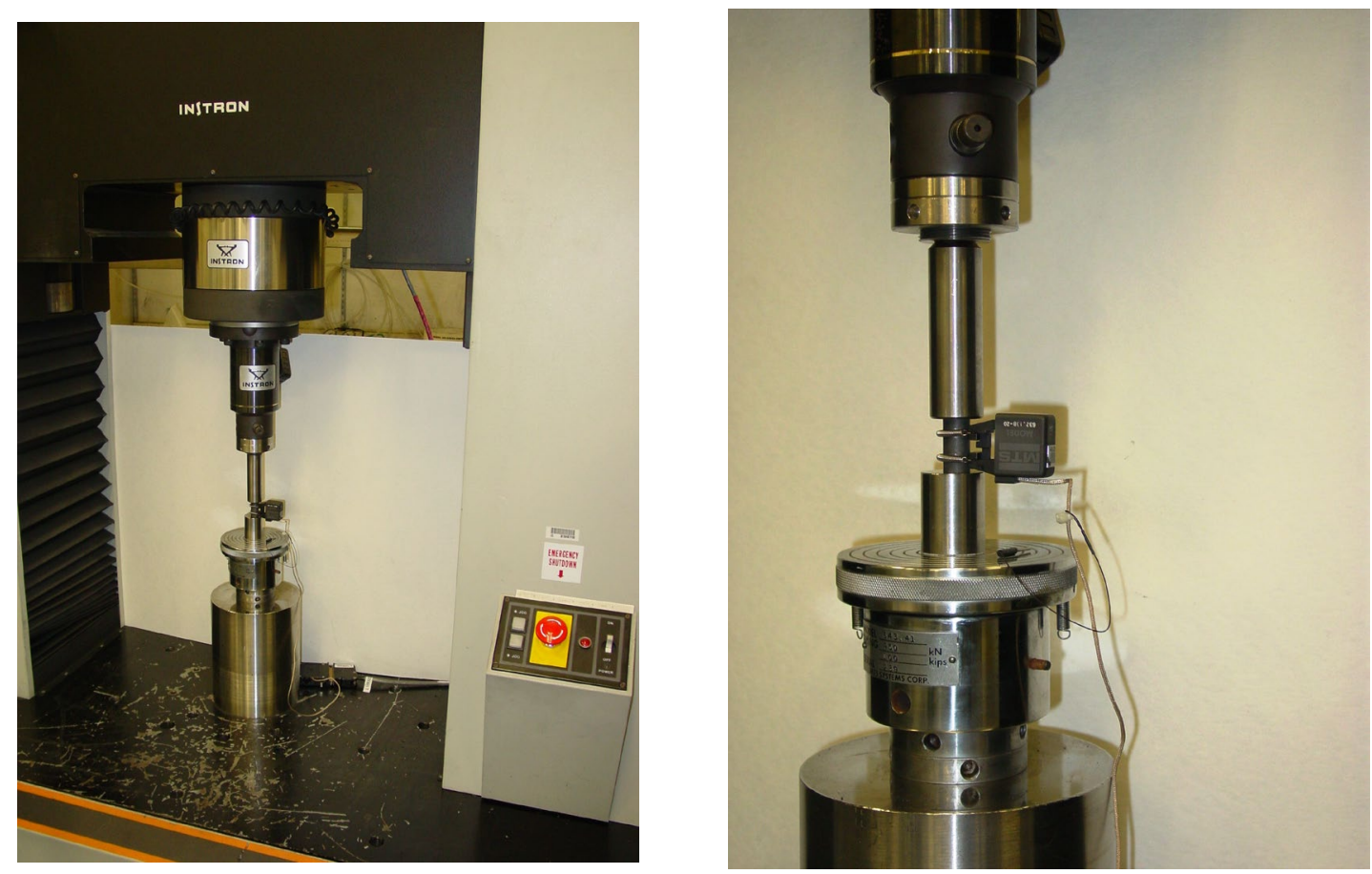

Figure 18 Instron apparatus used for compressive strength tests

Both "with grain" (PW) and "against grain" (PA) specimens were tested. According to ASTM C-695 and modification introduced by ASTM C-781, the diameter of test specimens for compressive strength measurements should be at least five times larger that the maximum grain size in graphite. The maximum grains size in PCEA graphite is $0.8 \mathrm{~mm}$. Therefore the "small" PCA specimens with diameter $12.7 \mathrm{~mm}$ were in compliance with this requirement. They also had the required aspect ratio of 2:1 between length and diameter. They exceeded the recommended minimum specimen size of $9.5 \mathrm{~mm}$ diameter and $19 \mathrm{~mm}$ length.

An Instron Model 4210 Electromechanical Test System was used for performing compressive strength tests. An Instron $50 \mathrm{kN}$ load cell, ID\# M216561 at a crosshead speed of $0.02 \mathrm{in} / \mathrm{min}$, and a MTS strain extensometer, Model 632.13B-20, ID\# M214476, 0.5" gage length were employed. This is a strain gauge type extensometer with knife blades that contact the specimen. The extensometer was attached to the specimen with springs. The specimen surface was locally sealed (approximately 0.2 in. diameter area on the specimen surface with a 5-minute epoxy) to prevent slippage of the extensometer blade without 
reinforcing specimen strength. The load and extensometer displacement data was recorded in text files via LabView software. The test specimens and the test data were archived for future examination.

\subsection{Compressive Strength Results}

The first tests with PA samples (orientation "against grain") were performed with the specimens as obtained after oxidation at 600 and $700{ }^{\circ} \mathrm{C}$. These tests have shown irregular load-strain relaxation curves after failure, very different from the shear fracture behavior characteristic to pristine (un-oxidized) samples (Figure 19). The fractured surface of oxidized specimens was in most cases aligned to the axis of the cylinder, sometimes bifurcated, and irregular in shape. After crushing, oxidized specimens produced dust and loose particles of different sizes (Fig. 19, b, c). In contrast, un-oxidized specimens showed shear type fractured surfaces, oriented at an angle of approximately $45^{\circ}$ from the cylinder axis (Fig. 19, a). The irregular fracture behavior of oxidized samples may be caused by uncontrolled failure under compression of the oxidized layer at the contact surface between the parallel faces of the cylinder and the Instron load cell.

In order to avoid this uncontrolled failure, all subsequent tests on oxidized specimens were made after machining of a $1 \mathrm{~mm}$ thick layer of oxidized material from both parallel faces of cylindrical samples. By doing that, we eliminated recording of relaxation after failure associated with the crushing of the oxidized layer on flat specimen surfaces in contact with the load cell. Examples of compression strength plots of PA specimens oxidized in similar conditions and end-faced after oxidation are shown in Fig. 19; they have the aspect characteristic typical to shear fracture of brittle materials. After test, the specimens showed typical shear aspect (Fig. 19, d, e) with fractured surfaces at a $45^{\circ}$ angle from the direction of load.

Figure 19 also shows that machining a thin $1 \mathrm{~mm}$ layer from both faces of oxidized specimens did not reduce the strength. All further tests on oxidized specimens in PW series (orientation with grain) were done after machining $1 \mathrm{~mm}$ from both faces. 


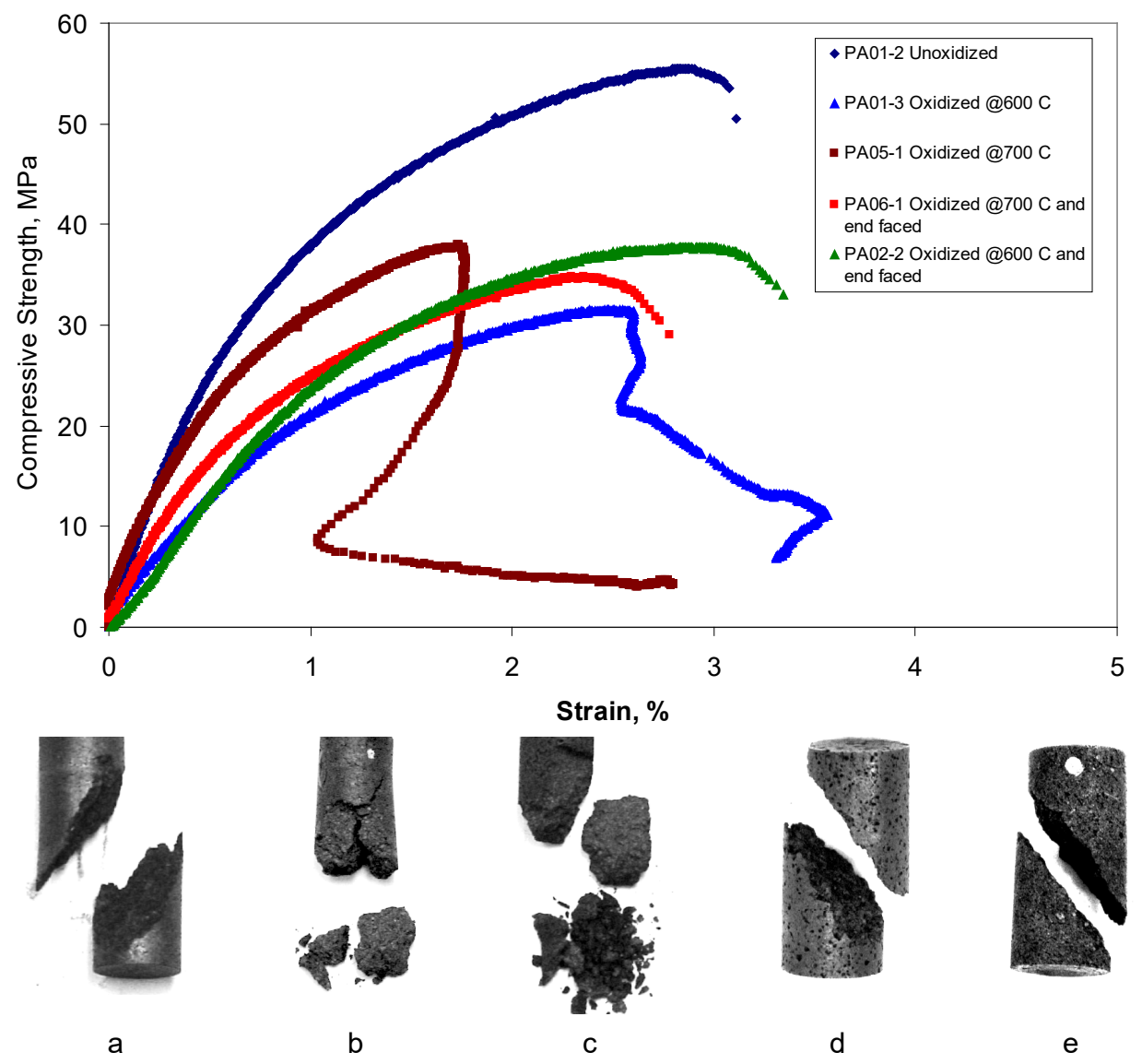

Figure 19 Compressive strength plots for pristine and oxidized PA specimens (against grain orientation), showing irregular relaxation curves and fracture aspect for "as oxidized" specimens, in contrast with shear fracture of un-oxidized sample and of oxidized specimens with $1 \mathrm{~mm}$ machined off from end faces. Specimen images: (a) PA01-2 un-oxidized; (b) PA 01-3, $11 \%$ weight loss at $600{ }^{\circ} \mathrm{C}$; (c) PA05-1, $12 \%$ weight loss at $700{ }^{\circ} \mathrm{C}$; (d) PA $02-2,11 \%$ weight loss at $600{ }^{\circ} \mathrm{C}$, end-faced; (e) PA 06-1, $12 \%$ weight loss at $700{ }^{\circ} \mathrm{C}$, end-faced

Table 5 reports average values of compressive strength and standard deviation for specimens in series PW and PA and the conditions of oxidation. In general there were 6 specimens tested for each oxidation condition and orientation. However, for the PA specimens, there were only 4 compressive tests done at each oxidation condition. The strength values reported for pristine (un-oxidized) specimens were measured in 2009 using same size PCEA graphite (with grains and against grains orientation) and on the same equipment. [29] 
Table 5 Results of compressive strength tests for "small" PCEA specimens (12.7 mm diameter) with two different orientations oxidized at 600 and $700{ }^{\circ} \mathrm{C}$

\begin{tabular}{|c|c|c|c|c|c|c|c|c|}
\hline \multirow[t]{3}{*}{ Orientation } & $\begin{array}{l}\text { Oxidation } \\
\text { temperature }\end{array}$ & $\begin{array}{l}\text { Weight } \\
\text { Loss }\end{array}$ & $\begin{array}{c}\text { Corrected } \\
\text { Weight } \\
\text { Loss }\end{array}$ & $\begin{array}{l}\text { Compressive } \\
\text { Strength }\end{array}$ & $\begin{array}{l}\text { Standard } \\
\text { deviation }\end{array}$ & $\begin{array}{l}\text { Strength } \\
\text { loss }\end{array}$ & $\begin{array}{l}\text { Strain } \\
\text { at max. } \\
\text { strength }\end{array}$ & $\begin{array}{l}\text { Number of } \\
\text { specimens } \\
\text { tested }\end{array}$ \\
\hline & \multicolumn{8}{|c|}{ Average values } \\
\hline & ${ }^{\circ} \mathrm{C}$ & $\%$ & $\%$ & $\mathrm{MPa}$ & $\mathrm{MPa}$ & $\%$ & $\%$ & \\
\hline \multirow{8}{*}{$\begin{array}{l}\text { PW series } \\
\text { (with grain) }\end{array}$} & pristine & 0 & 0 & 57.94 & 3.61 & 0.00 & & 12 \\
\hline & 600 & 5.50 & 5.60 & 48.82 & 0.75 & 15.75 & 2.43 & 6 \\
\hline & 600 & 10.18 & 10.43 & 42.75 & 2.78 & 26.22 & 2.02 & 4 \\
\hline & 600 & 15.57 & 15.53 & 33.74 & 3.50 & 41.76 & 2.02 & 6 \\
\hline & 700 & 0 & 0 & 57.94 & 3.61 & 0.00 & & 12 \\
\hline & 700 & 7.58 & 7.16 & 46.55 & 1.95 & 19.66 & 2.34 & 6 \\
\hline & 700 & 11.65 & 10.30 & 43.56 & 3.00 & 24.82 & 2.04 & 5 \\
\hline & 700 & 17.22 & 16.27 & 36.65 & 1.57 & 36.75 & 2.09 & 6 \\
\hline \multirow{4}{*}{$\begin{array}{c}\text { PA series } \\
\text { (against } \\
\text { grain) }\end{array}$} & pristine & 0 & 0 & 61.56 & 1.55 & 0.00 & & 12 \\
\hline & 600 & 10.25 & 10.84 & 29.31 & 7.90 & 52.39 & 3.04 & 4 \\
\hline & pristine & 0 & 0 & 61.56 & 1.55 & 0.00 & & 12 \\
\hline & 700 & 12.42 & 12.49 & 34.46 & 2.87 & 44.03 & 2.44 & 4 \\
\hline
\end{tabular}

The corrected weight loss $\alpha$ was defined as $\alpha=1-\rho / \rho_{\mathrm{o}}$, eq. (11), to account for any surface corrosion and weight loss that may have caused small changes in size (and thus in density, $\rho$ ) of oxidized specimens. [16] Table 6 shows that these corrections were more important for high oxidation levels at $700{ }^{\circ} \mathrm{C}$.

The test results on compressive strength are plotted in Figure 20 as a function of the corrected weight loss $\alpha$ at each oxidation temperature. Data obtained for samples in PW series are more statistically significant and cover three different levels of oxidation. Figure 20 shows two different fitting trends of these data: exponential (based on eq. (10) for Knudsen type decay) and linear. In the range of weight loss values covered by this report, both trends are predicted reasonably well, with the small difference that the $\mathrm{R}^{2}$ value of the exponential decay is higher than that of the linear trend at $600{ }^{\circ} \mathrm{C}$, and the other way around at $700{ }^{\circ} \mathrm{C}$. The following equations describe the trends:
$\sigma_{600}=57.94-1.5352 \alpha$
$\mathrm{R}^{2}=0.9966$
at $600{ }^{\circ} \mathrm{C}$
$\sigma 600=57.94 \exp (-0.329)$
$\mathrm{R}^{2}=0.9835$
at $600{ }^{\circ} \mathrm{C}$
$\sigma_{700}=57.94-1.365 \alpha$
$\mathrm{R}^{2}=0.9850$
at $700{ }^{\circ} \mathrm{C}$
$\sigma 700=57.94 \exp (-0.0283 \alpha)$
$\mathrm{R}^{2}=0.9971$
at $700{ }^{\circ} \mathrm{C}$ 
where $\sigma_{600}$ and $\sigma_{700}$ are compressive failure values for specimens oxidized at 600 and $700{ }^{\circ} \mathrm{C}$ and $\alpha$ is the density-corrected weight loss relative to the weight of the specimen before oxidation as defined in eq. (11).

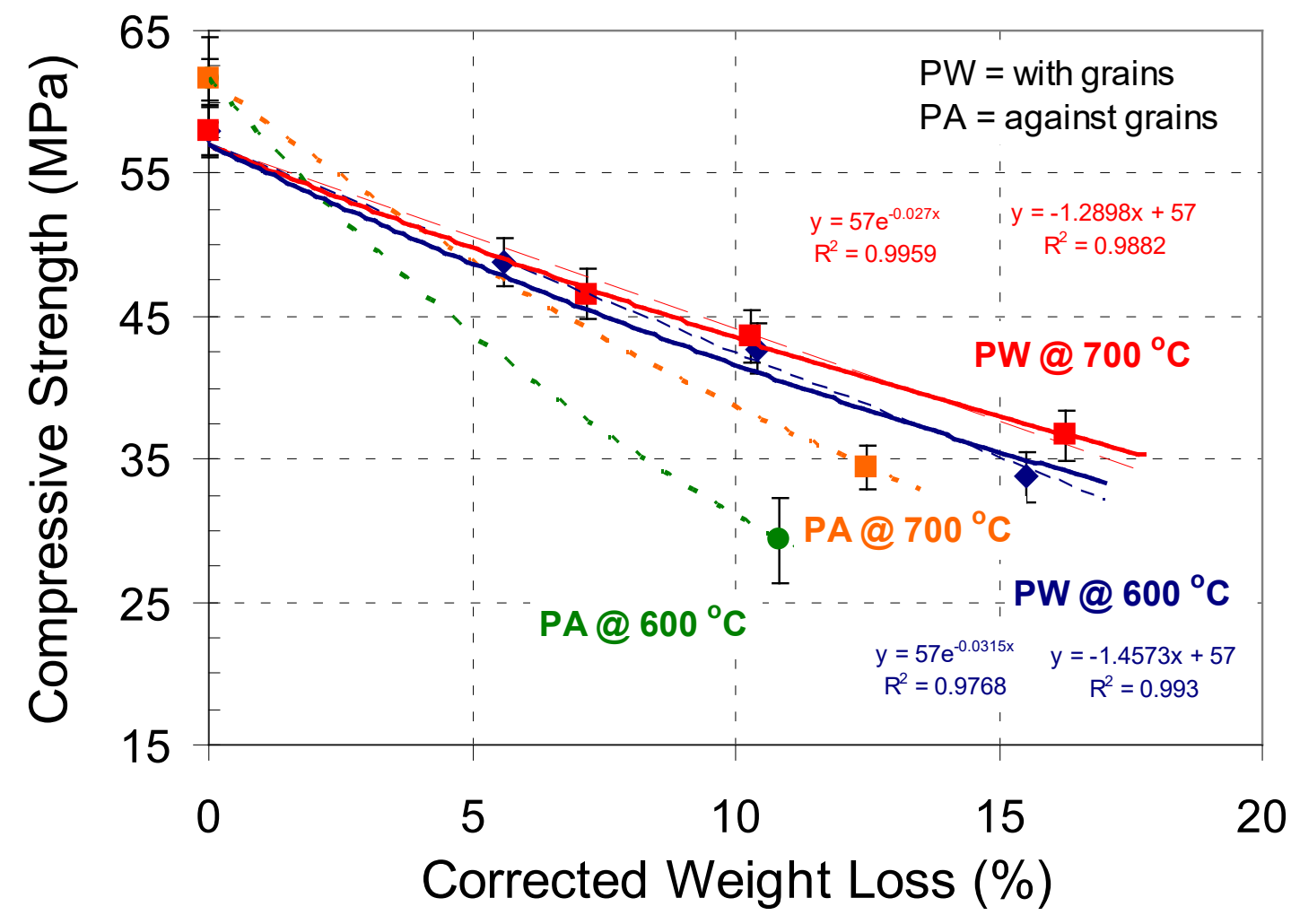

Figure 20 Average values for compressive strength to failure as a function of oxidative weight loss, oxidation temperature, and grain orientation of "small PCEA test specimens (12.7 mm diameter). Data from Table 5.

It is important to observe that oxidation at $600{ }^{\circ} \mathrm{C}$ causes more damage to compressive strength than oxidation at $700{ }^{\circ} \mathrm{C}$. This result is very clear for specimens in PW series, where data cover a broader range of oxidation levels. The rate of strength loss caused by oxidation was defined as the percentage drop of compressive strength, $\left(\sigma_{0}-\sigma_{\mathrm{T}}\right) / \sigma_{0}$, at given conditions (oxidative weight loss and temperature). Figure 21 shows the rate of strength loss versus effective (un-corrected) weight loss at two oxidation temperatures, for specimens of $\mathrm{PW}$ series. In the range of $15-16 \%$ weight loss, the rate of strength loss is $41.7 \%$ after oxidation at $600{ }^{\circ} \mathrm{C}$ and only $36.7 \%$ for oxidation at $700{ }^{\circ} \mathrm{C}$. The average rate of strength loss after oxidation at $600{ }^{\circ} \mathrm{C}$ is $2.65 \%$ of the initial compressive strength value for every $1 \%$ of weight loss; after oxidation at $700{ }^{\circ} \mathrm{C}$ the figure is only $2.35 \%$ of initial strength value per each percentage of weight loss. 


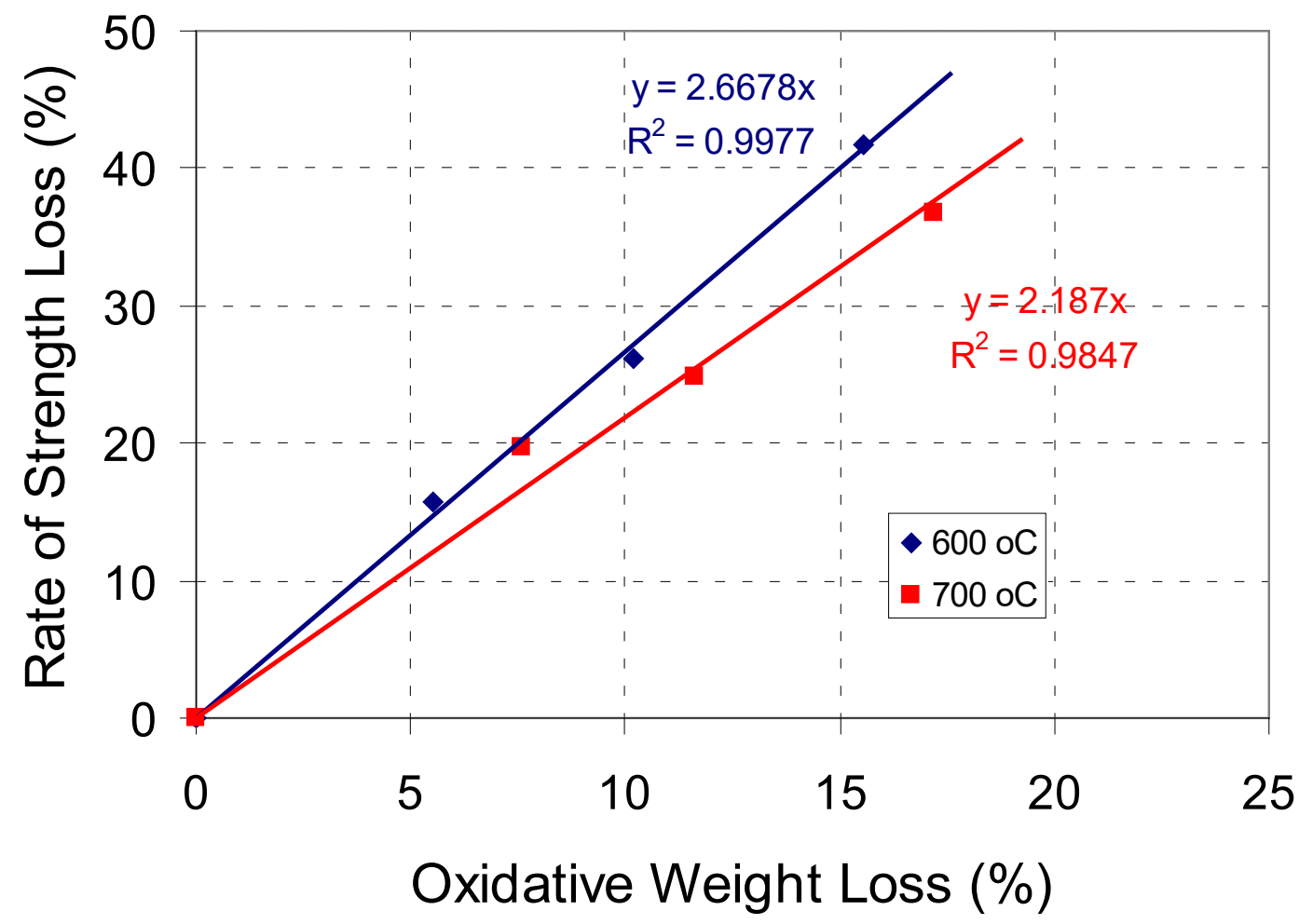

Figure 21 Rate of compressive strength loss versus weight loss by oxidation at 600 and $700{ }^{\circ} \mathrm{C}$ for "small" PW specimens (12.7 mm diameter) oriented "with grains". The lines represent best linear fitting of the data forced through origin. The slope quantifies the rate of strength loss (in percentage of compressive strength for un-oxidized specimen) for every $1 \%$ weight loss by oxidation. Data from Table 5. 


\section{RELATIONSHIP BETWEEN STRENGTH LOSS AND DISTRIBUTION OF OXIDATION LAYER}

The effect of the oxidation temperature on compressive strength values is related to the mechanism of development of porosity, and reflects the differences in the distribution of oxidation layer. In this section we attempt to connect information obtained from the two techniques used in this study: optical microscopy coupled with automatic image analysis and compressive strength measurements of oxidized graphite specimens

Specimens surface-oxidized at $700{ }^{\circ} \mathrm{C}$ are a special case because their oxidation layer is limited to a narrow zone, about $1.5 \mathrm{~mm}$ thick at the exposed external surface. This estimated is based on optical examination of oxidation profiles, see also Fig. 22 below. We attempted to develop a very simple mechanical model for analysis of the strength loss decay versus oxidation level. The goal is using available information (physical measurements, strength measurements, and structure of oxidized layer) to evaluate local properties in the oxidized layer (local strength and local density). If the model is correct, the relationship quantifying strength loss versus density decay should apply, as first introduced by Eto and Growcock [16], see eq. (6) above.

Surface-oxidized specimens can be viewed as a composite body, with an inner core of radius $r$ and an external annulus extending between $r$ and the outer specimen radius, $R$. It is assumed that the inner core is not modified by oxidation, and has the strength $\left(\sigma_{o}\right)$ and the density $\left(\rho_{\mathrm{o}}\right)$ characteristic to the un-oxidized material. It is also assumed that the external annulus is homogeneous and has lower strength and density than the un-oxidized material.

The measured compressive strength $\sigma$ is the ratio between the applied load, $F$, and the cross section of the specimen. [27] The latter is in fact the sum of base areas of the inner body and the annulus:

$$
\sigma=\frac{F}{\pi R^{2}}=\frac{F}{\pi r^{2}+\pi\left(R^{2}-r^{2}\right)}
$$

Knowing the compressive strength $\sigma(\mathrm{MPa})$ and the average radius of oxidized specimens, $R(\mathrm{~mm})$ one can calculate the average load force that would cause failure, $F(\mathrm{~N})$. It is assumed that the force to failure is distributed between the inner core and the annulus, and that the inner core component has the same compressive strength as the un-oxidized material $\sigma_{o}(\mathrm{MPa})$. The following equation is then used to estimate the compressive strength of the oxidized annulus, $\sigma_{x}(\mathrm{MPa})$, which is weakened by oxidization.

$$
F=\sigma_{o} \pi r^{2}+\sigma_{x} \pi\left(R^{2}-r^{2}\right)
$$

The volumes of annulus and core $\left(V_{a}\right.$ and $\left.V_{c}\right)$ and the specimen density before and after oxidation $\left(\rho_{o}\right.$ and $\rho$ ) are related through the following relationship for weight loss:

$$
\alpha=\frac{m_{o}-m}{m_{o}}=\frac{m_{o}-\left(m_{a}+m_{c}\right)}{m_{o}}=1-\frac{m_{a}}{m_{o}}-\frac{\rho_{o} V_{c}}{m_{o}}
$$


where it was assumed that the local density in the core equals the density of un-oxidized graphite $\left(m_{c}=\right.$ $\rho_{o} V_{c}$ ). With volumes in the core and annulus calculated from optical microscopy results and knowing the initial weight $\left(\mathrm{m}_{\mathrm{o}}\right)$ one can calculate the density in the annulus as a function of weight loss fraction and density of un-oxidized material:

$$
\rho_{a}=\frac{m_{o}(1-\alpha)-\rho_{o} V_{c}}{V_{a}}
$$

Table 6 shows the results of such an estimate for specimens oxidized at $700{ }^{\circ} \mathrm{C}$. The thickness of the oxidized layer $(\delta=R-r)$ was estimated from analysis of mosaic images shown above in Fig. 16 and of corresponding area histogram plots shown in Fig. 17.

Table 6 Estimated values of local density, local strength, and local burn-off in the oxidized layer (annulus) for specimens oxidized at $700{ }^{\circ} \mathrm{C}$ and three levels of burn-off, based on compressive strength tests and optical microscopy analysis of oxidized layer

\begin{tabular}{|c|c|c|c|c|c|c|c|c|}
\hline $\begin{array}{c}\text { Average } \\
\text { weight loss } \\
1-\mathrm{m} / \mathrm{m}_{\mathrm{o}}(\%)\end{array}$ & $\begin{array}{l}\text { Specimen } \\
\text { strength } \\
\sigma(\mathrm{MPa}) \\
\end{array}$ & $\begin{array}{c}\text { Load } \\
\text { force } \\
F \\
(\mathrm{~N}) \\
\end{array}$ & $\begin{array}{l}\text { Specimen } \\
\text { radius } \\
\mathrm{R}(\mathrm{mm}) \\
\end{array}$ & 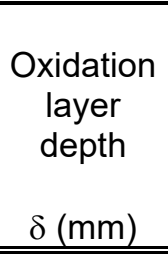 & $\begin{array}{c}\begin{array}{c}\text { Radius } \\
\text { of un- } \\
\text { oxidized } \\
\text { core }\end{array} \\
\mathrm{r}(\mathrm{mm}) \\
\end{array}$ & $\begin{array}{l}\text { Oxidized } \\
\text { annulus } \\
\text { strength } \\
\sigma_{x}(\mathrm{MPa}) \\
\end{array}$ & $\begin{array}{c}\text { Annulus } \\
\text { density } \\
\rho a \\
\left(\mathrm{~g} / \mathrm{cm}^{3}\right) \\
\end{array}$ & $\begin{array}{c}\text { Local } \\
\text { burn-off in } \\
\text { oxidized } \\
\text { layer } \\
1-\rho_{\mathrm{a}} / \rho_{\mathrm{o}}(\%) \\
\end{array}$ \\
\hline 7.6 & 46.5 & 6176 & 6.32 & 1.5 & 4.82 & 30.8 & 1.497 & 17.6 \\
\hline 11.6 & 43.6 & 5419 & 6.30 & 1.4 & 4.90 & 21.5 & 1.323 & 27.2 \\
\hline 17.2 & 36.6 & 4250 & 6.29 & 1.35 & 4.94 & 2.3 & 1.054 & 42.0 \\
\hline
\end{tabular}

Figure 22 shows detailed plots of area profile histograms for three specimens after oxidation at 700 ${ }^{\circ} \mathrm{C}$ at three levels of burn-off. The plots in this figure show details of oxidized layer near the surface of each specimen corresponding to East and West positions of the microscope field. The plots reproduce with more details area profile histograms from Fig. 17, bottom row. The thickness of oxidized layer determined from these plots has values between 1.35 and $1.50 \mathrm{~mm}$, with an uncertainty of $\pm 0.05 \mathrm{~mm}$. These values were used to calculate the local properties in oxidized layer. It was found that both the compressive strength and the local density drop severely, as shown in Table 6 . The local burn-off levels in the oxidized layer were calculated from density variation in the oxidized layer using eq. (11). 

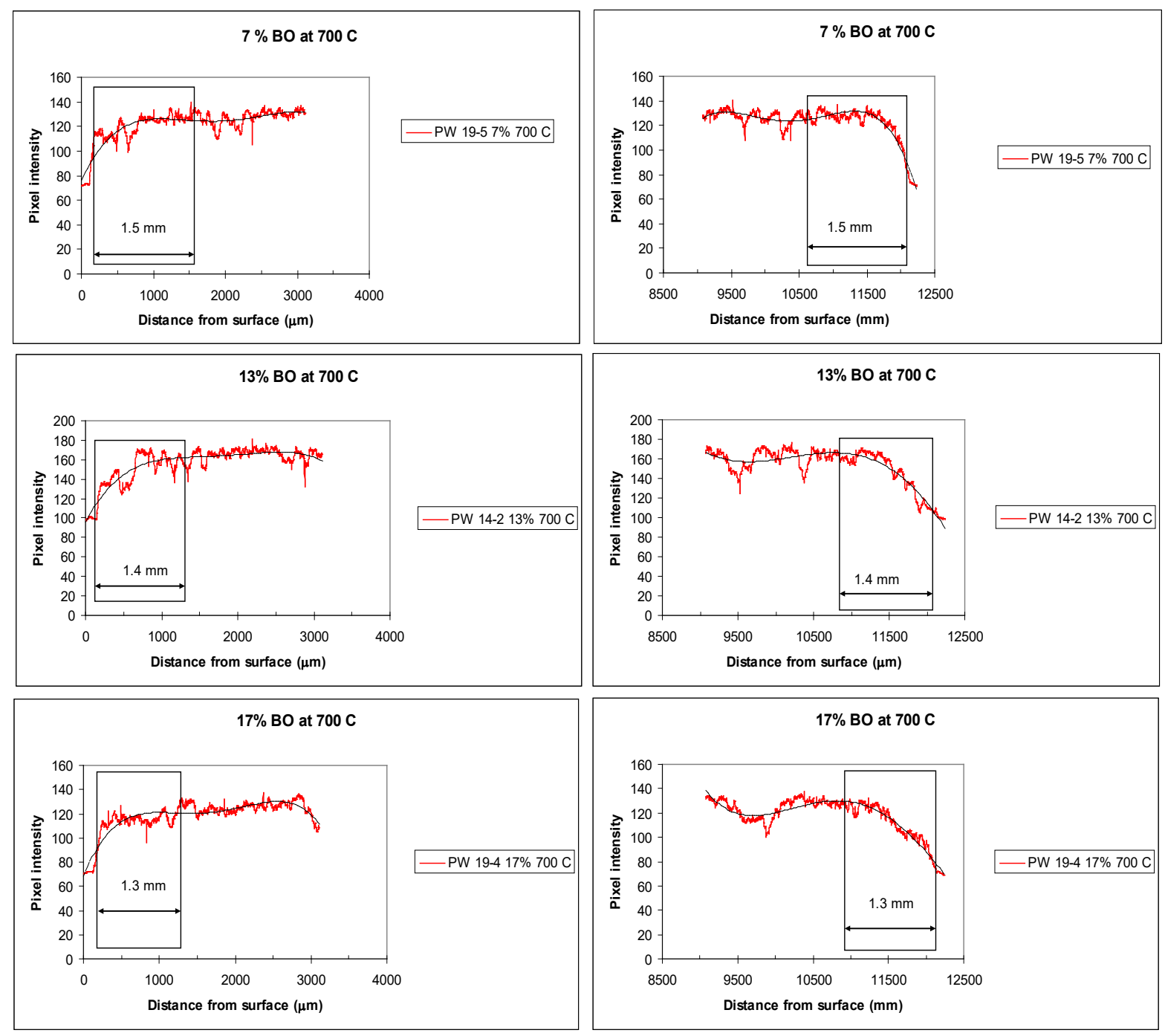

Figure 22 Detailed view and fit (using a $4^{\text {th }}$ grade polynomial) of area pixel intensity profiles for specimens oxidized at $700{ }^{\circ} \mathrm{C}$ to three levels of weigh loss. The samples analyzed here are the same as in the bottom row of Figure 17.

Although the model is grossly simplified, the results of this estimate follow the strength - density relationship proposed by Eto and Growcock [16] for homogeneously oxidized graphite specimens. Rewriting eq. (6) in logarithmic form and taking $m=1$ as proposed, the following relationship is obtained:

$$
\ln \left(\sigma / \sigma_{o}\right)=n \ln \left(\rho / \rho_{o}\right)
$$

Figure 23 shows the plot of relative strength $\left(\sigma / \sigma_{0}\right)$ versus relative density $\left(\rho / \rho_{0}\right)$ with the values from Table 6 , where $\sigma_{o}=57.94 \mathrm{MPa}$ and $\rho_{\mathrm{o}}=1.817 \mathrm{~g} / \mathrm{cm}^{3}$ are the compressive strength and the density of unoxidized specimens. 


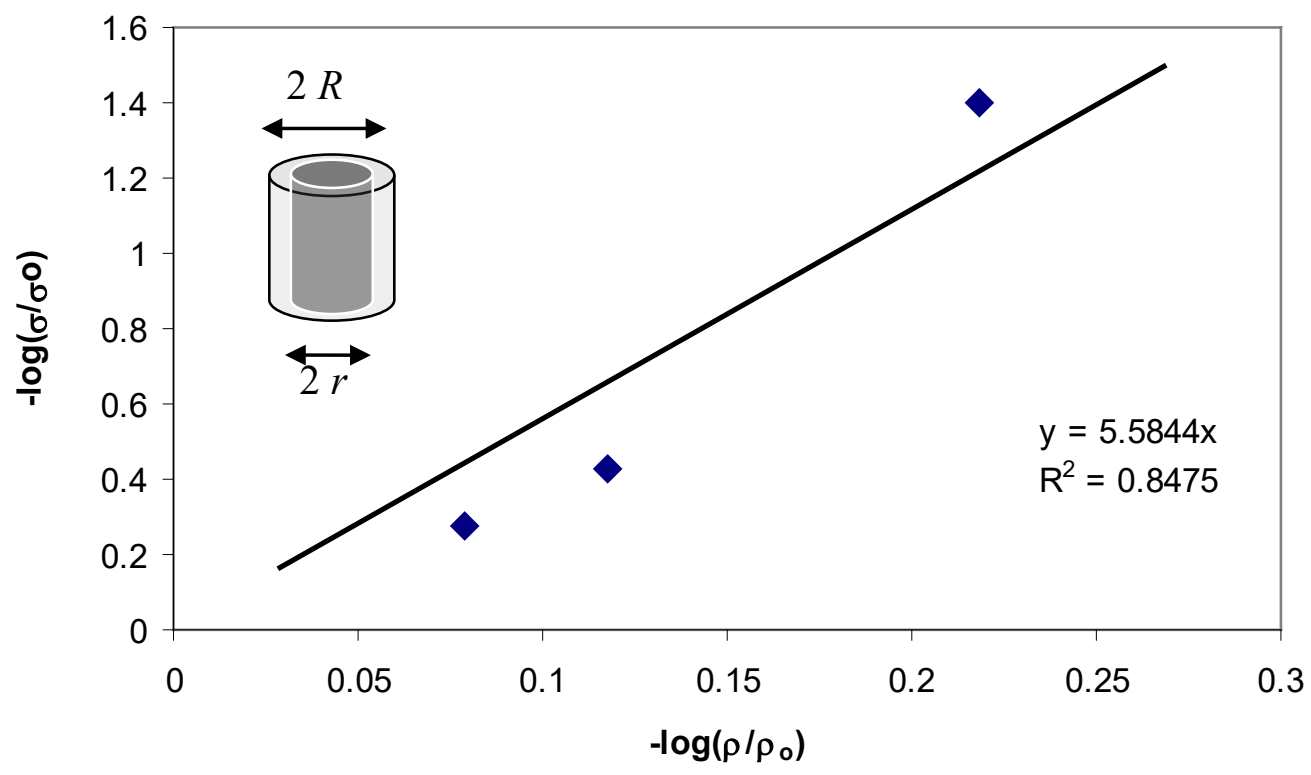

Figure 23 Verification of the empirical Eto-Growcock relationship [16] between strength loss and weight loss with local values in the oxidized layer, as obtained from the simplified mechanical model of strength-damaged annular cylinder containing an intact graphite core (inset).

Recall that the Eto-Growcock relationship was initially proposed for uniformly oxidized graphite specimens. In the model proposed here, the oxidized layer in the outer cylindrical annulus has been assumed to be uniform, as well. Figure 23 shows that the empirical trend proposed is verified with a reasonable correlation coefficient $\left(\mathrm{R}^{2}=0.85\right)$. The slope given by $n=5.6$ has a value comparable with other literature reports (between 4 and 10 in ref. [16]). Sensitivity analysis of the model has shown that the values estimated for local strength and local density in the oxidized layer depend slightly on the thickness values entered for the oxidized layer. The values measured by optical method have an uncertainty of about $5 \%$. There is a narrow optimum of thickness values which provide meaningful results (i.e. non-negative). This range is very close to the range of thickness values obtained from direct measurements. 


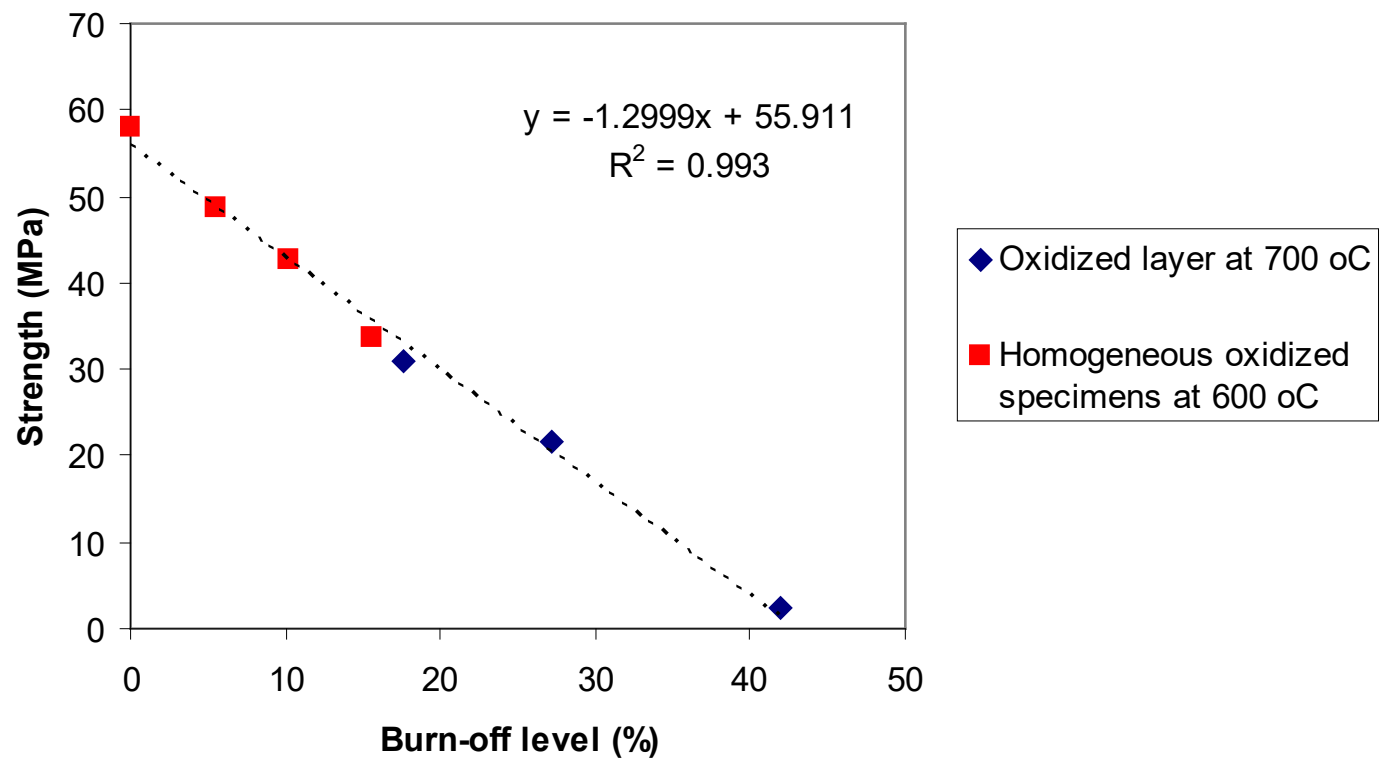

Figure 24 Comparison of local properties (strength and burn-off level) in oxidized layer estimated for specimens oxidized non-uniformly at $700{ }^{\circ} \mathrm{C}$ with average properties measured for uniformly oxidized specimens at $600{ }^{\circ} \mathrm{C}$. Data from Table 6 for local properties in oxidized layer are compared with data from Table 5 for average properties of homogeneously oxidized specimens.

The model does not apply to specimens oxidized at $600{ }^{\circ} \mathrm{C}$, which have uniform distribution of porosity. However, the local properties in the oxidized layer provided by the model are compatible with the average properties measured for whole specimens oxidized homogeneously at $600{ }^{\circ} \mathrm{C}$. Figure 24 shows strength versus burn-off data estimated for the oxidized layer of specimens oxidized at $700{ }^{\circ} \mathrm{C}$ (from Table 6) and actually measured strength versus weight loss results for PW specimens oxidized at $600{ }^{\circ} \mathrm{C}$ (from Table 5). Both series of data points are fitted by a straight line with correlation coefficient $\mathrm{R}^{2}=0.99$. The average rate of strength loss according to this correlation is $2.4 \%$ of un-oxidized compressive strength for each percentage of burn-off by oxidation. This value is very close to the rate of strength loss reported in Section 5.2 above for specimens oxidized at $600{ }^{\circ} \mathrm{C}(2.65 \%)$ and $700{ }^{\circ} \mathrm{C}(2.35$ $\%)$.

The trend shown in Figure 24 validates the model proposed above and provides credibility to the estimates of local properties in surface-limited oxidized layer. 


\section{ADDITIONAL NON-DESTRUCTIVE PROPERTIES EXAMINATION}

\subsection{Additional Properties Measurements}

Young's modulus, shear modulus, and Poisson's ratio were determined using the ultrasonic method for PA specimens prior and after oxidation.

Young's modulus $(E)$ was calculated from specimen's bulk density $(\rho)$ and the velocity of a longitudinal sound wave $(v)$ :

$$
E=\rho v^{2}
$$

Similarly, the shear modulus $(G)$ is calculated from the velocity of a shear sound wave $\left(v_{s}\right)$ and density $(\rho)$

$$
G=\rho\left(v_{s}\right)^{2}
$$

The velocity was calculated from measurements of time of flight of a sound wave over a known specimen length. The shear wave is typically measured with the probes in two orientations, and the two values are averaged. The apparatus was a Panametrics Squarewave pulse/received 5077 PD integrated with a National Instruments Dual Trace Oscilloscope Card model PXI-5122 and read with LabView software. The samples were coupled to the probes using ULTRAGEL II. The lab conditions were 22-25 ${ }^{\circ} \mathrm{C}, 55 \%$ humidity. The ultrasonic frequency was $2.25 \mathrm{MHz}$ and the associated wave length is $1.16 \mathrm{~mm}$. In previous experiments at ORNL it was verified that specimens as small as $6 \mathrm{~mm}$ thickness can be satisfactorily tested to determine the velocities and hence the elastic constants. More details on the methods used were presented in ref. [29].

\subsection{Effect of Oxidation on Young's Modulus and Shear Modulus}

Non-destructive examination of several additional properties was performed for "small" specimens in the WA series ("with grain") before and after oxidation at 600 and $700{ }^{\circ} \mathrm{C}$. The results obtained using the ultrasonic method are presented in Table 7.

Table 7 Effect of oxidation temperature on the density, Young's modulus and shear modulus

\begin{tabular}{|c|c|c|c|}
\hline & $\begin{array}{l}\text { Pristine PA } \\
\text { specimens }\end{array}$ & Oxidized at $600^{\circ} \mathrm{C}$ & Oxidized at $700^{\circ} \mathrm{C}$ \\
\hline Target weight loss (\%) & $\mathrm{n} / \mathrm{a}$ & 10 & 10 \\
\hline Actual weight loss (\%) & 0 & 10.25 & 12.42 \\
\hline Corrected weight loss (\%) & 0 & 10.84 & 12.49 \\
\hline Density $\left(\mathrm{g} / \mathrm{cm}^{3}\right)$ & 1.803 & 1.600 & 1.582 \\
\hline standard deviation $\left(\mathrm{g} / \mathrm{cm}^{3}\right)$ & 0.008 & 0.010 & 0.024 \\
\hline Young's modulus (GPa) & 9.37 & 6.69 & 5.16 \\
\hline standard deviation (GPa) & 0.22 & 0.34 & 0.88 \\
\hline Shear modulus (GPa) & 3.80 & 2.80 & 3.01 \\
\hline standard deviation (GPa) & 0.09 & 0.12 & 0.19 \\
\hline Number of specimens & 12 & 6 & 6 \\
\hline
\end{tabular}
of "small" PA specimens (12.7 mm diameter) with "against grain" orientation 
Both Young's and shear moduli decrease after oxidation. Although these results require more analysis, at this time it is interesting to observe the effect of oxidation temperature at comparable levels of weight loss $(10-12 \%)$. With the increase of temperature from 600 to $700{ }^{\circ} \mathrm{C}$, the Young's modulus decreases. In contrast, shear modulus has a less pronounced variation with temperature; the slight increase from 600 to $700{ }^{\circ} \mathrm{C}$ might be caused just by the statistical noise in the data. 


\section{CONCLUSION AND PERSPECTIVE}

In this report we have combined two different methods for analysis of pore development and mechanical properties variation induced by acute oxidation in air of nuclear-grade graphite (PCEA) which is a candidate material for NGNP.

We have expanded the analysis of oxidized layer by optical microscopy coupled with automated image analysis. Using standardized steps of image collection and processing, we demonstrated the usefulness of this simple but powerful technique. The technique provides information on the thickness of oxidized graphite layer and the properties of the pore system after oxidation. We have previously shown how oxidation generates new pores in the oxidized layer. Closer analysis this year has shown that the new pores are generated preferentially in the binder phase of graphite, and they have higher density in regions next to pre-existent pores. The latter serve as a channel for fast transport of oxidant species. When the supply of oxidant from the gas phase is not limited, the thickness of the oxidation layer seems to be affected in principal by the temperature of oxidation. Oxidation in isothermal conditions is accompanied by growth, merging, and finally collapse of pores in the oxidized layer. In a late stage the oxidized layer moves inwards, as the outer surface is consumed. The method described in this report makes it possible measuring the thickness of the oxidized layer, and is much faster than incremental machining, which is tedious and less accurate.

We combined optical analysis with compressive strength tests of oxidized specimens. To obtain good statistical reproducibility, the tests were repeated on large number of specimens oxidized at identical conditions. Two new series of PCEA samples were prepared oriented "with grain" (PW series) and "against grain" (PA series). Strength analysis focused on PW series. Samples oxidized in air at two temperatures $\left(600\right.$ and $\left.700{ }^{\circ} \mathrm{C}\right)$ and three levels of weight loss were obtained in this series. Oxidation of specimens in PA series was limited to one level of burn-off and two temperatures. Compressive strength measurements showed that oxidation at $600{ }^{\circ} \mathrm{C}$ is more damaging on strength than oxidation at $700{ }^{\circ} \mathrm{C}$, at comparable levels of weight loss. This conclusion is supported by measurements on both graphite orientations, and is rationalized by optical microscopy observations: Oxidation at $600{ }^{\circ} \mathrm{C}$ spreads uniformly in the bulk, while oxidation at $700{ }^{\circ} \mathrm{C}$ is limited to a narrow surface layer and bulk is apparently not affected. Comparing samples oxidized at 10-12\% weight loss, we also found that oxidation causes more strength loss on PA samples ("against grain") than on PW samples ("with grain").

Linking information provided by the two techniques, a simple model of non-uniformly oxidized specimens was developed. This original model provides reasonable estimates of the local strength, density, and level of burn-off in the oxidized layer for specimens with surface-limited penetration of oxidant. Moreover, comparing the estimates of local properties in the oxidized layer with average values measured for homogeneously oxidized specimens gave a consistent trend over a large range of variation burn-off levels (up to $40 \%$ ), thus providing credibility to the model advanced here.

Several non-destructive tests based on ultrasonic method were done with PA specimens before and after oxidation at two temperatures. The tests showed a continuous decrease of Young's modulus with the increase of oxidation temperature, and a lesser variation of shear modulus. These measurements should be regarded as a preliminary result which should be substantiated by more tests.

The need for obtaining more experimental data on the effect of pore development after thermal oxidation on the mechanical properties of graphite continues to be a pressing task of NGNP project. This is repeatedly emphasized in recent publications in the field $[9,10,11,30,36]$. The results shown in this report, as well as many other literature results, demonstrate that the properties of individual graphite grades determine their overall resistance in the VHTR environment. 
After more than six decades of research on nuclear graphite, the general mechanism of diffusionreaction controlled phenomena in heterogeneous systems (gas and microporous solids) are in general known. However, qualification of new grades of nuclear graphite for NGNP requires imperatively that graphite oxidation is considered along with all other property characterization (mechanical, thermal, irradiation behavior) as an important element for design and licensing. Detailed knowledge of reaction of new grades of graphite with oxidant gases that may be present in the helium coolant (water vapor, oxygen) is needed in order to model long-term behavior of graphite components in normal operating conditions. This knowledge is important for prediction of mechanical strength changes caused by development of porosity during oxidation, and for evaluation of integrity and lifetime of HGTR graphite components in real operation conditions. Alongside, the same type of information is needed for offnormal and transient conditions. Such studies are needed and must continue with determination of intrinsic kinetic constants for oxidation with low concentrations of water vapor and oxygen in helium and for characterization of effective diffusivity of these species in various grades of graphite. The database of results obtained will then be available for input in predictive models of density decay and strength loss variation as a function of temperature, concentration of oxidant impurities and duration of exposure, and spatial location in interior of graphite components. Such a predictive tool would be of very high value for the designer. 


\section{REFERENCES}

1 T. D. Burchell, Next Generation Nuclear Plant Phenomena Identification and Ranking Tables (PIRT), vol. 5. Graphite PIRTs, NUREG/CR-6944 vol. 5 (2007)

2 N. C. Gallego and T. D. Burchell, Comparison of NRC Graphite PIRT and DOE Planned Research Activities for Graphite, ORNL/NRC/LTR-09-01 (2009)

3 N. C. Gallego, T. D. Burchell, and M. Srinivasan, Milestone report on the "Workshop on Nuclear Graphite Research”, ORNL/NRC/LTR-09-03 (2009).

E. L. Fuller, J. M. Okoh, Kinetics and mechanisms of the reaction of air with nuclear grade graphites: IG-110, J Nucl Mater 240 (1997) 241

W.A. Propp, Graphite oxidation: Thermodynamics / reaction, DOE/SNF/REP-018, INEEL, Idaho Falls (Sept. 1998).

C. I. Contescu, S. Azad, D. Miller, M. J. Lance, F. S. Baker, Practical aspects for characterizing air oxidation of graphite, J Nucl Mater 381 (2008) 15-24

7 E. S. Kim, W. W. Lee, H. C. No, Analysis of geometrical effects on graphite oxidation through measurement of internal surface area, J Nucl Mater 348 (2006) 174-180

8 T. D. Burchell, A microstructurally based fracture model for polygranular graphites, Carbon 34 (1996) 297-316

9 T. D. Burchell, T. Yahr, R. Battiste, Modeling the multiaxial strength of H-451 nuclear grade graphite, Carbon 45 (2007) 2570-83.

10 L. Babout, P. M. Mummery, T. J. Marrow, A. Tzelepi, P. J. Withers, The effect of thermal oxidation on polycrystalline graphite studied by X-ray tomography, Carbon 43 (2005) 765774.

11 L. Babout, T. J. Marrow, P. M. Mummery, P.J. Withers, Mapping the evolution of density in 3D of thermally oxidized graphite for nuclear applications, Carbon 54 (2006) 829-834

12 N. Murdie, I. A. S. Edwards, H. Marsh, Changes in porosity of graphite caused by radiolytic gasification by carbon dioxide, Carbon 24 (1986) 267-275.

13 I M. Pickup, B. McEnaney, R. G. Cooke, Fracture processes in graphite and the effects of oxidation, Carbon 24 (1986) 535-543.

14 A. C. Collins, H. G. Masterson, P. P. Jennings, The effect of oxidation on the compressive strength of graphite, J. Nucl. Mater. 15 (1965) 134-136.

15 P. A. Thrower, J. C. Bognet, The effect of oxidation on the compressive strength of graphite, $13^{\text {th }}$ Biennial Conference Carbon, July 18-22, 1977, Irvine, CA.

16 M. Eto, F. B. Growcock, Effect of oxidizing environment on the strength of H451, PGX, and IG-11 graphites, Carbon 21 (1983) 135-147.

17 C. I. Contescu, T, Guldan, R. P. Wichner, T D. Burchell, Oxidation resistance of NGNP graphite materials, ORNL internal report, NGNP Program, Oak Ridge, TN (August 2008).

X. Luo, J. R. Robin, S Yu, Comparison of oxidation behaviors of different grades of nuclear graphite, Nucl. Sci. Engn. 151 (2005) 121-127

19 J. L. Wood, R. C. Bradt, P. L. Walker, Oxidation effects on toughness and slow crack growth in polycrystalline graphite, Carbon 18 (1980) 179-189. 

P. Ouagne, G. B. Neighbour, B. McEnany, Influence of oxidation on toughness parameters for two nuclear grade graphites, J. Phys. D. Appl. Phys. 38 (2005) 1259-1264. microstructure and the reduction of elastic modulus in thermally and radiolytically corroded nuclear graphites, Carbon 24 (1986) 545-549.

P. A. Thrower, D. R. Marx, The oxidation of graphite under compressive strength, Proc. $13^{\text {th }}$ Biennial Conference on Carbon, July 18-22, 1977, Irvine, CA (p. 41-42).

R. Krefeld, G. Linkenheil, Ph. Glaude, W. Karcher, Carbon '72 Conference, Baden-Baden, p. 216 (1972).

C. I. Contescu, T. D. Burchell, Characterization of porosity development in oxidized graphite using automated image analysis techniques, ORNL/TM-2009/192, Oak ridge, TN (September 2009)

26 "Standard test method for air oxidation of carbon and graphite in the kinetic regime", ASTM D 7542-09.

27 "Standard test method for compressive strength of carbon and graphite", ASTM C695-91 (Reapproved 2010)

C. I. Contescu, T. D. Burchell, ASTM activities on standard method for graphite oxidation, ORNL/GEN4/LTR-09-002, Oak Ridge, TN (August 2009)

T. D. Burchell, S D. Nunn, J. Strizak, M. Williams, AGC-1 Sister Specimen Testing Data Report, ORNL/TM-2009/025,Oak Ridge, TN (May, 2009)

R Moormann, H-J Hinssen, K Kuhn, Oxidation behavior of an HTR fuel element matrix graphite in oxygen compared to a standard nuclear graphite, Nucl. Eng. Design, 227 (2004) 281-284.

H-J Hinssen, K Kuhn, R Moormann, Oxidation experiments and theoretical examinations on graphite materials relevant for the PBMR, Proc. HTR2006, $3^{\text {rd }}$ International Topical Meeting on High Temperature Reactor Technology, Johannesburg, South Africa, October 1-4, 2006.

R. P. Wichner, S. J. Ball, T. D. Burchell, Potential damage to gas-cooled graphite reactors due to severe accidents, ORNL/TM-13661, Oak Ridge, TN (1999) acoustic microscopy and image analysis, J. Eng. Gas Turbine and Power, 131 (2009) 052904 graphite by oxygen and water vapor, J. Nucl. Mater. 393 (2009) 518-521

"Standard Practice for Testing of Graphite and Boronated Graphite Components for High Temperature Nuclear Reactors", ASTM C-781 